\title{
KREIN FORMULA AND $S$-MATRIX FOR EUCLIDEAN SURFACES WITH CONICAL SINGULARITIES
}

\author{
LUC HILLAIRET AND ALEXEY KOKOTOV
}

\begin{abstract}
Using the Krein formula for the difference of the resolvents of two self-adjoint extensions of a symmetric operator with finite deficiency indices, we establish a comparison formula for $\zeta$-regularized determinants of two self-adjoint extensions of the Laplace operator on a Euclidean surface with conical singularities (E. s. c. s.). The ratio of two determinants is expressed through the value $S(0)$ of the $S$-matrix, $S(\lambda)$, of the surface. We study the asymptotic behavior of the $S$-matrix, give an explicit expression for $S(0)$ relating it to the Bergman projective connection on the underlying compact Riemann surface and derive variational formulas for $S(\lambda)$ with respect to coordinates on the moduli space of E. s. c. s. with trivial holonomy.
\end{abstract}

\section{INTRODUCTION}

Spectral geometry aims at understanding the relations between the spectrum of some Laplace operator in a given geometrical setting and geometric properties of the latter. Polygons and polyhedra are among the simplest shapes one can consider and one could hope in this setting for a better understanding. This leads naturally to study the spectral geometry of Euclidean surfaces with conical singularities. Another motivation is the spectral theory of translation surfaces for which the geometric picture has many interesting developments (see [30] for instance).

One peculiarity of Laplacians on manifolds with conical points is that, due to the presence of conical points, a choice has to be made in order to get a self-adjoint operator. In this paper, we are interested in understanding how this choice affects several spectral quantities such as the resolvent and the zetaregularized determinant. Depending on the self-adjoint extension, this zeta-regularization procedure is not as straightforward as usual because of unusual behavior of the zeta function but it is still possible to define such a regularization (see [17, 12] and section [5.3) and we will prove a comparison formula for these determinants.

Comparison formulas for regularized determinants for conical manifolds were first found in [22] using a surgery formula à la BFK (see [5]) and in [17] using a contour integral method based on a secular equation that defines the spectrum. One of our motivations was to understand how the comparison formulas for different self-adjoint extensions from [22] read in the case of Euclidean surfaces with conical singularities and whether it is possible to express the determinants of the non Friedrichs selfadjoint extensions of the Laplacian on these surfaces through holomorphic invariants of the underlying Riemann surface (as it was done in [19] for the determinant of the Friedrichs extension). Indeed, Euclidean surfaces with conical singularities are our primary interest and we will restrict to this setting although many statements still make sense for more general conical manifolds.

It turns out that the geometric interpretation of the formulas obtained in [22] and [17] is not that straightforward and we have found it more convenient to establish the comparison formula for determinants using the Krein formula for the difference of resolvents of two self-adjoint extensions of a symmetric operator. We observe that the trace of the difference of two resolvents admits a nice representation through the so-called $S$-matrix of a Euclidean surface with conical singularities (E. s. c. s.) $X$. The latter matrix, or, more precisely, the meromorphic family of matrices $S(\lambda)$ is in some sense a characteristic feature of $X$. Indeed, we believe that some of the geometry of $X$ (such as for 
instance the lengths of saddle-connections between conical points- see Remark 4.3) is encoded in $S(\lambda)$ although it seems quite difficult to retrieve this kind of information. We should also remark that this $S$-matrix allows to write down a secular equation that can then be treated using the approach of [17] so that what we propose here may be seen as a geometric interpretation for the latter method. The comparison with [22] is less straightforward, it relies in interpreting the $S$-matrix as some kind of limiting Dirichlet-to-Neumann operator on a circle around the conical point when the radius of that circle goes to 0 . It can be noted here that, in contrast with [22] no extra condition is needed to obtain our formula.

We will thus prove the following theorem. The notion of regular self-adjoint extensions will be introduced in definition [5.2, section [5] and, for these self-adjoint extensions, the expression $P+Q S(0)$ makes sense (see remark [5.5).

Theorem 1. On a compact E.s.c.s. $X$, let $S(\lambda)$ be the $S$-matrix and $\Delta_{F}$ be the Friedrichs extension. Let $P$ and $Q$ be matrices that define a regular self-adjoint extension $\Delta_{L}$, and define

$$
D(\lambda):=\operatorname{det}(P+Q S(\lambda)) .
$$

Let $d$ be the dimension of $\operatorname{ker}(P+Q S(0))$ and let $D^{*}(0):=\lim _{\lambda \rightarrow 0}(-\lambda)^{-d} D(\lambda)$.

There exist $\alpha_{0}$ and $\Gamma$ such that the asymptotic expansion of $D(-|\lambda|)$ as $\lambda$ goes to $\infty$ is

$$
\ln D(-|\lambda|):=\alpha_{0} \ln (|\lambda|)+\Gamma+o(1) .
$$

The following identity then holds :

$$
\operatorname{det}_{\zeta}^{*}\left(\Delta_{L}\right)=\exp (-\Gamma) D^{*}(0) \operatorname{det}_{\zeta}^{*}\left(\Delta_{F}\right)
$$

in which $\operatorname{det}_{\zeta}^{*}$ is the modified zeta-regularized determinant (see definition 5.14.)

To fulfil our second aim we then need to understand more explicitly what kind of geometric information is encoded in the family $S(\lambda)$. We focus on the limiting behavior when the spectral parameter goes to 0 since this is the regime that comes up in the comparison formula. We will prove that most of the matrix elements in this limit have an interpretation through values of the Bergman projective connection and the basic holomorphic differentials taken at the conical point in the corresponding distinguished holomorphic local parameter (see section 4.3). Since we expect translation surfaces to have particular and interesting features, we will also say a word on the $S$-matrix on these special kind of surfaces. Namely, we will derive variational formulas for the $S$-matrix when it is differentiated with respect to moduli parameters. These results answer most of the questions which motivated our study.

Organization of the paper. In the small second section we will recall the basic facts about Euclidean surfaces with conical singularities. We will in particular recall that these can be viewed as Riemann surfaces with flat conformal conical metric.

In section 3 we recall some basic properties of the Friedrichs Laplace operator on E. s. c. s., and introduce the object of our primary interest - the $S$-matrix; we also derive here the standard formula for the derivative of the $S$-matrix with respect to $\lambda$.

In section 4 we study the asymptotic behavior of $S(\lambda)$ as $\lambda$ goes to $-\infty$ and find the geometric interpretation of $S(0)$. We also also apply the variational formulas of [19] to obtain the variations of $S(\lambda)$ with respect to moduli parameters on translation surfaces.

In section 5 we study various self-adjoint extensions of the Laplace operator on E. s. c. s. and prove the comparison formula for their $\zeta$-regularized determinants.

\section{Acknowledgements.}

The research of LH was partly supported by the ANR programs NONaa and Teichmüller.

The research of AK was supported by NSERC. AK thanks Hausdorff Research Institute for Mathematics (Bonn) and Laboratoire de Mathématiques Jean Leray (Nantes) for hospitality. AK also thanks the MATPYL program for supporting his coming and stay in Nantes where this research began. 
We acknowledge useful conversations with G. Carron and with D. Korotkin whose advice in particular helped us to simplify some constructions from section 4.2.

\section{EUCLIDEAN SURFACES WITH CONICAL SINGULARITIES}

2.1. Euclidean surfaces with conical singularities as Riemann surfaces with conformal flat conical metrics. A Euclidean surface with conical singularities (E. s. c. s.) is a compact (orientable) surface glued from Euclidean triangles. One can take as an example of such a surface the boundary of a connected but not necessarily simply connected polyhedron in $\mathbb{R}^{3}$.

When two triangles are glued together and after rotating one of the triangles around the common edge we observe that the intrinsic geometry of the surface is locally that of the plane. There, the surface actually is smooth and equipped with a smooth Euclidean metric. At a vertex $p$ where $k$ triangles with angles $\vartheta_{1}, \ldots, \vartheta_{k}$ are glued together, the surface is locally isometric to a neighbourhood of the tip of the Euclidean cone of total angle $\theta_{p}=\vartheta_{1}+\cdots+\vartheta_{k}$. The surface $X$ is thus equipped with a Euclidean metric that is smooth except at the vertices $p$ for which $\theta_{p} \neq 2 \pi$.

It follows for instance from 28 , that $X$ can be provided with a complex analytic structure becoming a compact Riemann surface $\tilde{X}$; moreover, the usual Euclidean metric on $X$ gives rise to a flat conformal (i. e. defining the same complex structure) metric on $\tilde{X}$. Abusing notations slightly, from now on we won't make any difference between $X$ and $\tilde{X}$.

On the other hand, consider a flat conformal metric $m$ with conical singularities on a Riemann surface $X$. In a vicinity of a conical point $p, m$ can be written as

$$
m=|g(z)||z|^{2 b}|d z|^{2},
$$

where $z$ is a holomorphic local parameter near $p, z(p)=0, b>-1$ and $g(z)$ is a holomorphic function of the local parameter such that $g(0) \neq 0$.

It is shown in [28] that one can choose a holomorphic change of variables $z=z(\zeta)$ such that

$$
|g(z(\zeta))||z(\zeta)|^{2 b}\left|z^{\prime}(\zeta)\right|^{2}=|\zeta|^{2 b}
$$

and, therefore,

$$
m=|\zeta|^{2 b}|d \zeta|^{2}
$$

in the local parameter $\zeta$. This means that the Riemannian surface $(X, m)$ near $p$ is isometric to the standard Euclidean cone of angle $2 \pi(b+1)$. Troyanov [28] showed that the Riemannian manifold $(X, m)$ can be triangulated in such a way that all the conical points will be among the vertices of the triangulation meaning thus that $(X, m)$ is an E. s. c. s.

Definition 1. Let $X$ be a compact Riemann surface with conformal flat conical metric (i. e. a E. s. c. s.) and let $p \in X$ be a conical point. Then any holomorphic local parameter $\zeta$ in which the metric takes the form (2.2) is called distinguished.

Notation : We will denote by $P$ the set of conical points and by $X_{0}:=X \backslash P$ the complement of $P$ in $X$. We set $M:=\operatorname{Card}(P)$ the number of conical points. At each $p \in P$, the total cone angle is denoted by $\theta_{p}$.

2.1.1. Translation and half-translation surfaces. A translation (resp. half-translation) surface is a E. s. c. s. that has trivial holonomy (resp. holonomy group $\mathbb{Z}_{2}$ ). These are important examples of E.s.c.s. with very nice geometric properties (see [30] for a survey on these).

Translation surfaces are Riemann surfaces $X$ that are equipped with a conformal flat conical metric given by the modulus square, $m=|\omega|^{2}$, of a holomorphic 1-form (an Abelian differential) $\omega$. If $P$ is a zero of $\omega$ of multiplicity $k$ then $p$ is a conical point of the translation surface $X$ with conical angle $2 \pi(k+1)$. The moduli space $H_{g}$ of pairs $(X, \omega)$ (where $X$ is a compact Riemann surface of genus $g \geq 1$, 
$\omega$ is a holomorphic 1-form on $X$ ) is stratified according to the multiplicities of the zeros of the 1-form $\omega$. Denote by $H_{g}\left(k_{1}, \ldots, k_{M}\right)$ the stratum consisting of pairs $(X, \omega)$, where $\omega$ has $M$ zeros, $p_{1}, \ldots, p_{M}$ of multiplicities $k_{1}, \ldots, k_{M}$ (according to Riemann-Roch theorem one has $k_{1}+\cdots+k_{M}=2 g-2$ ). The stratum $H_{g}\left(k_{1}, \ldots, k_{M}\right)$ is a complex orbifold of dimension $2 g+M-1$.

Let $(X, \omega) \in H_{g}\left(k_{1}, \ldots, k_{M}\right)$. Choose a canonical basis of cycles $\left\{a_{\alpha}, b_{\alpha}\right\}$ on the Riemann surface $X$ and take $M-1$ contours $\gamma_{k}, k=2, \ldots, M$ on $X$ connecting $p_{1}$ with $p_{2}, \ldots, p_{M}$

The local coordinates on $H_{g}\left(k_{1}, \ldots, k_{M}\right)$ (which are called Kontsevich-Zorich homological coordinates, see [20]) are given by the following integrals:

$$
\begin{gathered}
A_{\alpha}=\oint_{a_{\alpha}} \omega ; \quad \alpha=1, \ldots, g, \\
B_{\alpha}=\oint_{b_{\alpha}} \omega ; \quad \alpha=1, \ldots, g, \\
z_{k}=\int_{\gamma_{k}} \omega ; \quad k=2, \ldots, M-1 .
\end{gathered}
$$

A half-translation surface is a compact Riemann surface with flat conical metric $m=|q|$, where $q$ is a meromorphic quadratic differential with at most simple poles.

Example 2.1. Consider the Riemann sphere $\mathbb{C} P^{1}$ with metric

$$
\frac{|z|^{2}|d z|^{2}}{\prod_{k=1}^{6}\left|z-z_{k}\right|},
$$

where $z_{k} \in \mathbb{C}, z_{k} \neq 0$ and $z_{i} \neq z_{k}$ if $i \neq k$. This is a half-translation surface with 7 conical points $0, z_{1}, \ldots, z_{6}$. The conical angle at 0 is $4 \pi$, the conical angles at each point $z_{k}$ are equal to $\pi$.

Such a surface can be viewed by considering a Euclidean pair of pants (with one $4 \pi$ singularity) and by sewing each leg and the waist with itself (thus creating the six $\pi$ singularities).

\section{The Friedrichs LAPlacian AND the S-MATRIX}

Let $X$ be a compact E.s.c.s.. In this section we will recall the definition of the Friedrichs Laplacian associated with the (singular) metric and define the so-called $S$-matrix. We will then collect several properties of this matrix.

We denote by $\Delta$ the minimal closed extension of the Euclidean Laplacian defined on $\mathcal{C}_{0}^{\infty}\left(X_{0}\right)$, and by $\Delta^{*}$ its adjoint with respect to the Euclidean $L^{2}$ scalar product

$$
\langle u, v\rangle:=\int_{X} u \bar{v} d x
$$

Near each conical point $p$, any $u \in \operatorname{dom}\left(\Delta^{*}\right)$ has the following asymptotic behavior in polar coordinates $(r, \theta)$ (see, e. g., [23], [22], [24] or [19]):

$$
u(r, \theta)=\sqrt{2 \theta_{p}}\left(a_{0}^{+}+a_{0}^{-} \ln (r)\right)+\sum_{\nu} \sqrt{2|\nu| \theta_{p}}\left(a_{\nu}^{+} r^{|\nu|}+a_{\nu}^{-} r^{-|\nu|}\right) \exp (i \nu \theta)+u_{0},
$$

where $\nu$ ranges over $N_{p}:=\left\{\frac{2 \pi}{\theta_{p}} \cdot k,|k \in \mathbb{Z} \backslash\{0\}| k \mid,<\frac{\theta_{p}}{2 \pi}\right\}$, and $u_{0} \in \operatorname{dom}(\Delta)$.

Notation : We will denote by $N=\cup_{p \in P} N_{p}$, and we will abusively still denote by $\nu$ an element of $N$. Choosing an element $\nu$ of $N$ thus amounts to choosing a conical point $p$ and then some $\nu$ in $N_{p}$. Unless needed we will omit the reference to $p$. The square roots prefactor in (3.1) are just normalization constants. We will denote these constants by $C_{0}:=\sqrt{2 \theta_{p}}$ and $C_{\nu}:=\sqrt{2|\nu| \theta_{p}}$ (we recall that since $\nu$ implicitly depends on $p$, so does $C_{\nu}$ ). 
In the distinguished local parameter $\zeta$ near $p$ we have, for $\nu=\frac{2 \pi}{\theta_{p}} \cdot k$

$$
\begin{gathered}
\zeta^{k}=r^{\nu} \exp (i \nu \theta)= \begin{cases}r^{|\nu|} \exp (i \nu \theta) & \text { if } \nu>0, \\
r^{-|\nu|} \exp (i \nu \theta) & \text { if } \nu<0 .\end{cases} \\
\bar{\zeta}^{-k}=r^{-\nu} \exp (i \nu \theta)= \begin{cases}r^{|\nu|} \exp (i \nu \theta) & \text { if } \nu<0, \\
r^{-|\nu|} \exp (i \nu \theta) & \text { if } \nu>0 .\end{cases}
\end{gathered}
$$

Thus the asymptotic expansion (3.1) may also be written

$$
u(\zeta, \bar{\zeta})=C_{0}\left(a_{0}^{+}+a_{0}^{-} \ln (|\zeta|)\right)+\sum_{k=1}^{\frac{\theta_{p}}{2 \pi}-1} C_{k \frac{2 \pi}{\theta_{p}}}\left(a_{k}^{+} \zeta^{k}+a_{k}^{-} \bar{\zeta}^{-k}+a_{-k}^{+} \bar{\zeta}^{k}+a_{-k}^{-} \zeta^{-k}\right)+u_{0} .
$$

A straightforward application of Green's formula (combined with the choice of the normalization constants $\left.C_{0}, C_{\nu}\right)$ then implies that, for any $u, v$ in $\operatorname{dom}\left(\Delta^{*}\right)$,

$$
\left\langle\Delta^{*} u, v\right\rangle-\left\langle u, \Delta^{*} v\right\rangle=\sum_{p \in P}\left[a_{0}^{+} \cdot \overline{b_{0}^{-}}-a_{0}^{-} \cdot \overline{b_{0}^{+}}+\sum_{\nu \in N_{p}}\left(a_{\nu}^{+} \cdot \overline{b_{\nu}^{-}}-a_{\nu}^{-} \cdot \overline{b_{\nu}^{+}}\right)\right]
$$

where the $a_{\nu}^{ \pm}$are the coefficients in the expansion of $u$ and the $b_{\nu}^{ \pm}$those in the expansion of $v$.

Setting $\mathcal{G}(u, v):=\left\langle\Delta^{*} u, v\right\rangle-\left\langle u, \Delta^{*} v\right\rangle$ we define a Hermitian symplectic form on $\operatorname{dom}\left(\Delta^{*}\right) / \operatorname{dom}(\Delta)$ whose lagrangian subspaces parametrize the self-adjoint extensions of $\Delta$.

3.1. The Friedrichs extension. For any $u \in \operatorname{dom}(\Delta)$ a straightforward integration by parts gives

$$
\langle\Delta u, u\rangle=\int_{X}|\nabla u|^{2} d x
$$

so that the Friedrichs procedure (see [4] section 10.3 or [26] theorem X.23) provides us with a selfadjoint extension that we denote by $\Delta_{F}$. Since a function $u$ in $\operatorname{dom}\left(\Delta_{F}\right)$ is characterized by $\nabla u \in$ $L^{2}(X)$, we obtain the following lemma.

Lemma 3.1. The lagrangian subspace in $\operatorname{dom}\left(\Delta^{*}\right) / \operatorname{dom}(\Delta)$ that corresponds to the Friedrichs extension is

$$
\left\{a_{\nu}^{-}=0\right\} .
$$

Definition 2. We denote by $H^{s}:=\operatorname{dom}\left(\Delta_{F}^{\frac{s}{2}}\right)$ the scale of Sobolev spaces associated with it. In particular we set $\operatorname{dom}\left(\Delta_{F}\right):=H^{2}$.

Remark 1. This definition of $H^{s}$ is not completely standard. In particular, because of the conical singularities, for $m>1$ the following inclusion is strict (see [11] for a much more detailed discussion about this fact) :

$$
\left\{u \in L^{2}|\forall| \alpha \mid \leq m, \partial^{\alpha} u \in L^{2}\right\} \subset H^{m} .
$$

By standard spectral theory, the resolvent of $\Delta_{F}$ defines a continuous operator from $H^{s}$ to $H^{s+2}$. We also recall that since $X$ is compact, the Rellich-type injection theorem from [8] implies that $\Delta_{F}$ has compact resolvent so that the spectrum is non-negative and discrete. 
3.2. The S-matrix. We will now define a matrix associated to the flat structure and to the choice of the Friedrichs extension.

First, for any $\nu$, we fix $F_{\nu}=C_{\nu} r^{-|\nu|} \exp (i \nu \theta) \rho(r)$ where $\rho$ is some fixed cut-off function that is identically 1 near the corresponding conical point $p$.

We define $\Lambda_{\nu}$ to be the linear functional on $H^{2}$ satisfying

$$
\forall u \in H^{2}, \Lambda_{\nu}(u)=\mathcal{G}\left(u, F_{\nu}\right)
$$

We have the following lemma.

Lemma 3.2. The linear functional $\Lambda_{\nu}$ is continuous on $H^{2}$ and

$$
\forall u \in H^{2}, \Lambda_{\nu}(u)=a_{\nu}^{+}
$$

where $a_{\nu}^{+}$is the coefficient in the expansion (3.1) of $u$ near $p$.

Proof. The fact that $F_{\nu} \in \operatorname{dom}\left(\Delta^{*}\right)$ implies that $\Lambda_{\nu}$ is indeed continuous. The second statement follows from the respective asymptotic behaviors of $F_{\nu}$ and $u$ near $p$.

Remark 3.3. The preceding lemma in particular implies that the linear functional $\Lambda_{\nu}$ doesn't depend on the choice of the cut-off function $\rho$.

For $\lambda \in \mathbb{C} \backslash[0, \infty)$, we set

$$
G_{\nu}(\cdot ; \lambda):=\left(\Delta_{F}-\lambda\right)^{-1} \Lambda_{\nu}
$$

Since $\Lambda_{\nu}$ is in $H^{-2}, G_{\nu}$ is in $L^{2}$, and for any $u \in H^{2}$, we have

$$
\Lambda_{\nu}(u)=\left\langle\left(\Delta_{F}-\lambda\right) u, G_{\nu}(\cdot ; \bar{\lambda})\right\rangle .
$$

Since the resolvent is analytic in $\lambda, G_{\nu}(\cdot ; \lambda)$ defines an analytic family of $L^{2}$ functions.

Observe that the latter equation is equivalent to

$$
\left(\Delta^{*}-\lambda\right) G_{\nu}(\cdot ; \lambda)=0,
$$

so that $G_{\nu}(\cdot ; \lambda) \in \operatorname{dom}\left(\Delta^{*}\right)$. Moreover, by testing against an appropriate $u \in H^{2}$ we can compute the coefficients $a_{\mu}^{-}$of $G_{\nu}$. This yields $a_{\mu}^{-}=\delta_{\mu \nu}$ (where $\delta$ is the Kronecker symbol).

The following proposition gives a formula for $G_{\nu}$

\section{Proposition 3.4.}

For any $\lambda \in \mathbb{C} \backslash[0, \infty)$, set $f_{\nu}(\cdot ; \lambda):=\left(\Delta^{*}-\lambda\right) F_{\nu}$ and $g_{\nu}(\cdot ; \lambda):=-\left(\Delta_{F}-\lambda\right)^{-1} f_{\nu}(\cdot ; \lambda)$. Then $g_{\nu}(\cdot ; \lambda)$ is an analytic family in $H^{2}$ and

$$
G_{\nu}(\cdot ; \lambda)=F_{\nu}(\cdot)+g_{\nu}(\cdot ; \lambda) .
$$

Proof. Computation shows that $f_{\nu}$ is in $L^{2}(X)$ which yields that $g_{\nu}$ is in $H^{2}$ since $\lambda$ is in the resolvent set of $\Delta_{F}$. Since $f_{\nu}$ and the resolvent depend analytically on $\lambda$ so does $g_{\nu}$. By construction, $\left(\Delta^{*}-\lambda\right)\left(F_{\nu}+g_{\nu}\right)=0$ and all the $a_{\mu}^{-}$coefficients of $G_{\nu}-\left(F_{\nu}+g_{\nu}\right)$ vanish. This means that the latter function is in $H^{2}$ and thus is 0 since $\lambda$ is in the resolvent set.

Example 3.5. Let us consider the complete cone $[0, \infty) \times \mathbb{R} / \alpha \mathbb{Z}$. Using separation of variables we have that $G_{\nu}(r, \theta ; \lambda)=k(r) \exp (i \nu \theta)$. For $\nu \neq 0$, by definition $k$ is the unique solution to

$$
-k^{\prime \prime}-\frac{1}{r} k^{\prime}+\left(\frac{\nu^{2}}{r^{2}}-\lambda\right) k=0
$$

which is $L^{2}(r d r)$ and asymptotic to $C_{\nu} r^{-|\nu|}$ near 0 . Thus $k$ is proportional to $K_{\nu}(\sqrt{-\lambda} r)$ where $K_{\nu}$ is Bessel-MacDonald function (see [25] for instance). For $\nu=0$, the singular behavior is logarithmic but $k(r)$ still is proportional to $K_{0}(\sqrt{-\lambda} r)$ 
Definition 3.6 (The S-matrix). We define the S-matrix $S(\lambda)$ by

$$
S_{\mu \nu}(\lambda)=\Lambda_{\mu}\left(g_{\nu}(\cdot ; \lambda)\right)
$$

Remark 3.7. Alternatively, $S_{\mu \nu}(\lambda)$ is the $a_{\mu}^{+}$coefficient of $g_{\nu}(\cdot ; \lambda)$. It is also the $a_{\mu}^{+}$coefficient of $G_{\nu}(\cdot ; \lambda)$. Observe that the entries of the $S$-matrix are numbered by non-integer numbers.

Using (3.7), we have the following alternative expression

$$
S_{\mu \nu}(\lambda)=\left\langle\left(\Delta_{F}-\lambda\right) g_{\nu}(\cdot ; \lambda), G_{\mu}(\cdot ; \bar{\lambda})\right\rangle=\left\langle f_{\nu}(\cdot ; \lambda), G_{\mu}(\cdot ; \bar{\lambda})\right\rangle
$$

It follows from the analyticity of $g_{\nu}$ that $S(\lambda)$ is analytic on $\mathbb{C} \backslash[0 ; \infty)$.

Example 3.8. We define $S_{\alpha}(\lambda)$ to be the S-matrix of the cone of angle $\alpha$. According to example 3.5, $S_{\alpha}(\lambda)$ is diagonal. Moreover, the asymptotic expansion of Bessel-Macdonald functions near 0 is

$$
\begin{gathered}
K_{0}(z)=-\ln (z)+\ln (2)-\gamma+o(1), \\
K_{|\nu|}(z)=\frac{\pi}{2 \sin (|\nu| \pi)}\left[\frac{z^{-|\nu|}}{2^{-|\nu|} \Gamma(1-|\nu|)}-\frac{z^{|\nu|}}{2^{|\nu|} \Gamma(1+|\nu|)}+O\left(z^{2-|\nu|}\right)\right]
\end{gathered}
$$

where $\Gamma$ is Euler gamma function and $\gamma$ Euler's constant (see for instance [25]). This yields

$$
\begin{gathered}
{\left[S_{\alpha}(\lambda)\right]_{00}=\ln (\sqrt{-\lambda})-(\ln (2)-\gamma),} \\
{\left[S_{\alpha}(\lambda)\right]_{\nu \nu}=-\frac{\Gamma(1-|\nu|)(-\lambda)^{|\nu|}}{2^{2|\nu|} \Gamma(1+|\nu|)} .}
\end{gathered}
$$

The interpretation of $S(\lambda)$ is given by the following lemma.

Lemma 3.9. For any $\lambda \in \mathbb{C} \backslash[0, \infty)$ and any $F \in \operatorname{ker}\left(\Delta^{*}-\lambda\right)$. Denote by $A^{ \pm}(F)$ the vector consisting of all the coefficients $a_{\nu}^{-}$(resp. $a_{\nu}^{+}$) of $F$. Then we have

$$
A^{+}=S(\lambda) A^{-} \text {. }
$$

Remark 3.10. Interpreting $A^{-}$as some kind of incoming data and $A^{+}$as the outgoing data justifies the interpretation of the $S$-matrix as a scattering matrix.

Proof. Set $\tilde{F}:=\sum_{\nu} a_{\nu}^{-} G_{\nu}(\cdot ; \lambda)$ then $F-\tilde{F}$ is in $\operatorname{dom}\left(\operatorname{ker}\left(\Delta^{*}-\lambda\right)\right)$. Since all the $a_{\nu}^{-}$vanish, $F-\tilde{F}$ actually is in $\operatorname{dom}\left(\Delta_{F}\right)$. This implies $F=\tilde{F}$ since $\lambda$ is in the resolvent set of $\Delta_{F}$. Writing each $G_{\nu}=F_{\nu}+g_{\nu}$, we obtain :

$$
a_{\mu}^{+}=\Lambda_{\mu}\left(\sum_{\nu} a_{\nu}^{-} g_{\nu}\right)=\sum_{\nu} S(\lambda)_{\mu \nu} a_{\nu}^{-}
$$

Remark 3.11. Until now we haven't used the fact that the underlying metric actually is Euclidean with conical singularities. The preceding construction is fairly general and can be made on any manifold with conical singularities. Actually, it can be done in an abstract manner for any symmetric operator with (equal) finite deficiency indices (compare with section 13.4 of [14]).

Before coming to the main aim of this paper, which is to understand how much geometric information is contained in the S-matrix, we derive first two basic properties of $S_{\mu \nu}(\lambda)$. 
3.3. Derivative of the S-matrix. In this section a dot will mean differentiation with respect to $\lambda$, and we prove the following lemma.

Lemma 3.12. On $\mathbb{C} \backslash[0, \infty)$, we have

$$
\dot{S}_{\mu \nu}=\left\langle G_{\nu}(\cdot ; \lambda), G_{\mu}(\cdot ; \bar{\lambda})\right\rangle
$$

Proof. We start from the relation

$$
\left(\Delta_{F}-\lambda\right) g_{\nu}(\cdot ; \lambda)=-\Delta^{*} F_{\nu}(\cdot)+\lambda F_{\nu}(\cdot)
$$

that we differentiate with respect to $\lambda$. Since $F_{\nu}$ doesn't depend on $\lambda$ and $g_{\nu}$ is analytic in $H^{2}$ we obtain

$$
\left(\Delta_{F}-\lambda\right) \dot{g}_{\nu}(\cdot ; \lambda)=F_{\nu}(\cdot)+g_{\nu}(\cdot ; \lambda)=G_{\nu}(\cdot ; \lambda)
$$

This gives

$$
\begin{aligned}
\dot{S}(\lambda)_{\mu \nu} & =\Lambda_{\mu}\left(\dot{g}_{\nu}(\cdot ; \lambda)\right) \\
& =\Lambda_{\mu}\left(\left(\Delta_{F}-\lambda\right)^{-1} G_{\nu}(\cdot ; \lambda)\right) \\
& =\left\langle G_{\nu}(\cdot ; \lambda), G_{\mu}(\cdot ; \bar{\lambda})\right\rangle,
\end{aligned}
$$

where we have used (3.7) for the last identity.

3.4. Relation with the resolvent kernel. Denote by $R\left(x, x^{\prime} ; \lambda\right)$ the resolvent kernel of the Friedrichs extension $\Delta_{F}$.

Fix $x^{\prime} \in X_{0}$. As a function of the first argument, $R\left(\cdot, x^{\prime} ; \lambda\right)$ is locally in $H^{2}$ near each conical point $p$. Thus according to (3.1), there exists a collection $a_{\nu}^{+}\left(x^{\prime} ; \lambda\right)$ such that, in the neighbourhood of $p$ we have the following asymptotic expansion :

$$
R\left(r \exp (i \theta), x^{\prime} ; \lambda\right)=\sum_{\nu \in N_{p}} C_{\nu} a_{\nu}^{+}\left(x^{\prime} ; \lambda\right) r^{|\nu|} \exp (i \nu \theta)+r_{0}
$$

with $r_{0} \in \overline{\mathcal{C}_{0}^{\infty}\left(X_{0}\right)} H^{2}$.

Using (3.6), we see that $a_{\nu}^{+}\left(x^{\prime} ; \lambda\right)=\mathcal{G}\left(R\left(\cdot, x^{\prime} ; \lambda\right), F_{\nu}\right)$ and thus, the former expansion may be differentiated with respect to $x^{\prime}$ in any compact set of $X_{0}$.

The following proposition makes the relation between $a_{\nu}^{+}\left(x^{\prime} ; \lambda\right)$ and $G_{\nu}\left(x^{\prime} ; \lambda\right)$ more explicit.

Proposition 3.13. For any $x^{\prime} \in X_{0}$, we have

$$
G_{\nu}\left(x^{\prime} ; \lambda\right)=a_{\nu}^{+}\left(x^{\prime} ; \lambda\right)
$$

where $a_{\nu}^{+}\left(x^{\prime} ; \lambda\right)$ is the previously described coefficient in the asymptotic expansion of $R\left(\cdot, x^{\prime} ; \lambda\right)$ near $p$.

In other words, $G_{\nu}\left(x^{\prime} ; \lambda\right)$ is obtained by selecting in the resolvent kernel $R\left(x, x^{\prime} ; \lambda\right)$ some particular term in the asymptotic behavior $x \rightarrow p$. Using $\overline{R\left(x^{\prime}, x ; \bar{\lambda}\right)}=R\left(x, x^{\prime} ; \lambda\right)$ there are similar statements when we fix $x$ and let $x^{\prime}$ tends to $p$.

Proof. Denote by $\Delta_{1}$ the Euclidean Laplace operator on $\mathcal{C}_{0}^{\infty}\left(X \backslash\left(P \cup\left\{x^{\prime}\right\}\right)\right)$. This operator fits in the general theory described in section 3 by considering that $x^{\prime}$ actually is the vertex of a cone of angle $2 \pi$. In particular, Green's formula (3.1) is still valid provided we take into account log singularities at $x^{\prime}$. The resolvent kernel $R\left(\cdot, x^{\prime} ; \lambda\right)$ and $G_{\nu}(\cdot ; \lambda)$ both belong to $\operatorname{dom}\left(\Delta_{1}^{*}\right)$. the singularities of $R$ are described by the functions $a_{\nu}^{+}$near the conical points and $R$ has a log singularity near $x^{\prime}$ whereas $G_{\nu}$ is smooth near $x^{\prime}$ and its singular behavior near the conical points $G_{\nu}$ is prescribed by (3.10). Green's formula thus yields :

$$
\left\langle\left(\Delta_{1}^{*}-\lambda\right) R\left(\cdot, x^{\prime} ; \lambda\right), G_{\nu}(\cdot \bar{\lambda})\right\rangle-\left\langle R\left(\cdot, x^{\prime} ; \lambda\right),\left(\Delta_{1}^{*}-\bar{\lambda}\right) G_{\nu}\left(x^{\prime} ; \bar{\lambda}\right)\right\rangle=\overline{G_{\nu}\left(x^{\prime} ; \bar{\lambda}\right)}-a_{\nu}^{+}\left(x^{\prime} ; \lambda\right) .
$$


Since $\left(\Delta_{1}^{*}-\lambda\right) R\left(\cdot, x^{\prime} ; \lambda\right)=0=\left(\Delta_{1}^{*}-\bar{\lambda}\right) G_{\nu}\left(x^{\prime} ; \bar{\lambda}\right)$, we obtain

$$
\overline{G_{\nu}\left(x^{\prime} ; \bar{\lambda}\right)}=a_{\nu}^{+}\left(x^{\prime} ; \lambda\right) \text {. }
$$

We now use the fact that $G_{\nu}(x ; \lambda)$ is analytic for $\lambda \in \mathbb{C} \backslash[0, \infty)$ and real for real (and negative) $\lambda$. Thus by analytic continuation

$$
\overline{G_{\nu}\left(x^{\prime} ; \bar{\lambda}\right)}=G_{\nu}\left(x^{\prime}, \lambda\right)
$$

\section{The S-MATRIX OF E.S.C.S.}

In this section we try to understand what kind of geometric information is encoded in the S-matrix of a Euclidean surface with conical singularities. We begin by studying the asymptotic behavior of $S(\lambda)$ as $\lambda$ goes to $-\infty$.

4.1. $S(-|\lambda|)$ for large $\lambda$. It is a general fact that the behavior of the resolvent kernel when $\lambda$ goes to $-\infty$ is a local quantity.

This is confirmed by the following lemma.

Lemma 4.1. When $\lambda$ goes to $\infty$ then

$$
[S(-|\lambda|)]_{\mu \nu}=O\left(|\lambda|^{-\infty}\right),
$$

if $\mu$ and $\nu$ do not correspond to the same conical point.

When $\mu$ and $\nu$ correspond to the same conical point $p$ of angle $\alpha$ then we have

$$
[S(-|\lambda|)]_{\mu \nu}=\left[S_{\alpha}(-|\lambda|)\right]_{\mu \nu}+O\left(|\lambda|^{-\infty}\right),
$$

where $S_{\alpha}$ denotes the $S$-matrix on the infinite cone of total angle $\alpha$.

Moreover both identities may be differentiated with respect to $\lambda$.

Proof. We use the representation of the resolvent kernel using the heat kernel (that we denote here by $\left.\mathcal{P}\left(t, x, x^{\prime}\right)\right)$ :

$$
R\left(x, x^{\prime} ;-|\lambda|\right)=\int_{0}^{\infty} \exp (-t|\lambda|) \mathcal{P}\left(t, x, x^{\prime}\right) d t
$$

We now use a standard construction of a parametrix for the heat kernel (see [7] for instance). We first enumerate the set of conical points writing $P:=\left\{p_{i}, 1 \leq p_{i} \leq M\right\}$. Then, for each $p_{i}$ we choose $\tilde{\chi}_{i}$ and $\chi_{i}$ two smooth cut-off functions such that $\operatorname{supp}\left(\chi_{i}\right) \subset\left\{\tilde{\chi}_{i}=1\right\}, \chi_{i}$ is identically 1 near $p$ and $X$ is isometric to a neighbourhood of the tip of the cone of angle $\theta_{p_{i}}$ on the support of $\tilde{\chi}_{i}$. We complete the collections $\left(\chi_{i}\right)_{i \leq M}$ and $\left(\tilde{\chi}_{i}\right)_{i \leq M}$ to $\left(\chi_{i}\right)_{i \leq \tilde{M}},\left(\tilde{\chi}_{i}\right)_{i \leq \tilde{M}}$ in such a way that $\left(\chi_{i}\right)_{i \leq \tilde{M}}$ is a partition of unity, $\tilde{\chi}_{i}$ is identically 1 on the support of $\chi_{i}$ and, for $M<i \leq \tilde{M}, X$ is isometric to a neighbourhood of the origin in $\mathbb{R}^{2}$ on the support of $\tilde{\chi}_{i}$. We also set $\mathcal{P}_{i}$ to be the heat kernel on the cone corresponding to $p_{i}$ if $i \leq M$ and on the plane otherwise and define

$$
\tilde{\mathcal{P}}\left(t, x, x^{\prime}\right)=\sum_{i=1}^{\tilde{M}} \tilde{\chi}_{i}(x) \mathcal{P}_{i}\left(t, x, x^{\prime}\right) \chi_{i}(x) .
$$

Using Duhamel's principle and the fact that $\mathcal{P}_{i}$ fastly decays away of the diagonal (see eq (1.1) of 7]) yields that $\tilde{\mathcal{P}}(t)-\mathcal{P}(t)$ maps $L^{2}$ into $H^{s}$ for any $s$, and

$$
\|\tilde{\mathcal{P}}(t)-\mathcal{P}(t)\|_{L^{2} \rightarrow H^{s}}=O\left(t^{\infty}\right)
$$

when $t$ goes to 0 , so that $\tilde{\mathcal{P}}$ is a parametrix for the heat kernel. 
Inserting into (4.1) and integrating against $f_{\nu}$ we obtain

$$
g_{\nu}(x ;-|\lambda|)=\tilde{\chi}_{i}(x) \int_{0}^{\infty} \int_{X} \mathcal{P}_{i}\left(t, x, x^{\prime}\right) f_{\nu}\left(x^{\prime} ;-|\lambda|\right) d S\left(x^{\prime}\right) d t+r_{\lambda}(x),
$$

where the remainder $r_{\lambda} \in H^{2}$ and $\left\|r_{\lambda}\right\|_{H^{2}}=O\left(|\lambda|^{-\infty}\right)$ and the index $i$ corresponds to the conical point corresponding to $\nu$. The first statement follows. The second also follows by remarking that $F_{\nu}, f_{\nu}$ and $\Lambda_{\nu}$ can also be seen as living on the cone and that the latter equation is also valid on the complete cone. Differentiating with respect to $\lambda$ amounts to replace $\mathcal{P}$ by $\Delta_{F} \mathcal{P}$ and we can use the same argument.

Using example 3.8 we obtain the following proposition as a corollary.

Proposition 4.2. When $\lambda$ goes to $\infty$ we have

$$
\begin{aligned}
& {[S(-|\lambda|)]_{\mu \nu}=O\left(|\lambda|^{-\infty}\right) \text { if } \mu \neq \nu,} \\
& {[S(-|\lambda|)]_{\nu \nu}=-\frac{\Gamma(1-|\nu|)}{2^{2|\nu|} \Gamma(1+|\nu|)} \cdot|\lambda|^{|\nu|}+O\left(|\lambda|^{-\infty}\right), \text { if } \nu \neq 0,} \\
& {[S(-|\lambda|)]_{00}=\frac{1}{2} \ln (|\lambda|)-(\ln (2)-\gamma)+O\left(|\lambda|^{-\infty}\right) .}
\end{aligned}
$$

Remark 4.3. It would be interesting to study the asymptotic behavior of $S( \pm i|\lambda|)$. It is then expected to see contributions of periodic diffractive orbits (compare with [16]).

4.2. Explicit formulas for $S(0)$. In this subsection we will show that for $\nu \neq 0$ the coefficient $S_{\mu \nu}(\lambda)$ is continuous at $\lambda=0$ and may be expressed using standard objects of the Riemannian surface $X$.

Recall that, in the distinguished local parameter $\zeta$ near some conical point $P$ the asymptotic expansion was given in (3.4). It follows that we have

$$
\begin{cases}F_{\nu}(\zeta, \bar{\zeta}) \sim C_{\nu} \zeta^{-k} & k>0 \\ F_{\nu}(\zeta, \bar{\zeta}) \sim C_{\nu} \bar{\zeta}^{k} & k<0\end{cases}
$$

where, as usual $\nu$ and $k$ are related by the relation $\nu=\frac{2 \pi}{\theta_{p}} \cdot k$.

We first prove the following lemma.

Lemma 4.4. If $\nu \neq 0$ then $G_{\nu}(\cdot ; \lambda)$ is continuous at $\lambda=0$ and $G_{\nu}(\cdot ; 0)$ is a harmonic $L^{2}$ function on $X$ such that

$$
\left\{\begin{aligned}
G_{\nu}(\zeta, \bar{\zeta} ; 0) & =\zeta^{-k}+O(1) & k>0 \\
G_{\nu}(\zeta, \bar{\zeta} ; 0) & =\bar{\zeta}^{k}+O(1) & k<0
\end{aligned}\right.
$$

Proof. Recall that we have set $G_{\nu}(\cdot ; \lambda)=F_{\nu}+g_{\nu}(\cdot ; \lambda)$, where $g_{\nu}(\cdot ; \lambda)$ is the unique solution to

$$
\left(\Delta_{F}-\lambda\right) g_{\nu}(\cdot ; \lambda)=-\left(\Delta^{*}-\lambda\right) F_{\nu} .
$$

Since $\int_{X}\left(\Delta^{*}-\lambda\right) F_{\nu} d x=0$ the continuity at 0 follows from the fact that the $\operatorname{ker}\left(\Delta_{F}\right)$ consists only in the constant function. By continuity we obtain $G_{\nu}(\cdot ; 0)$ is a solution to $\Delta^{*} G_{\nu}(\cdot ; 0)=0$ and, therefore, $G_{\nu}(\cdot ; 0)$ is harmonic on $X_{0}$.

Remark 4.5. Let $\zeta$ be denoting the distinguished local parameter near a fixed $p \in P$. The problems

$$
\left\{\begin{array}{l}
\Delta U_{k}=0 \quad \text { on } \quad X \backslash P \\
U_{k} \sim \zeta^{-k}+O(1), \text { as } \zeta \rightarrow 0
\end{array}\right.
$$

for $0<k<\frac{\theta_{p}}{2 \pi}$ and 


$$
\left\{\begin{array}{l}
\Delta U_{k}=0 \text { on } \quad X \backslash P \\
U_{k} \sim \bar{\zeta}^{k}+O(1), \text { as } \zeta \rightarrow 0
\end{array}\right.
$$

for $-\frac{\theta_{p}}{2 \pi}<k<0$ have solutions only up to an additive constant. On the other hand, the problem

$$
\left\{\begin{array}{l}
\Delta u=0 \quad \text { on } \quad X \backslash P \\
u \sim \log r+O(1), \text { as } \zeta \rightarrow 0
\end{array}\right.
$$

doesn't have a solution. Thus the behaviour of the coefficients $S_{0 \nu}(\lambda)$ and $S_{\mu 0}(\lambda)$ may not even be properly defined for $\lambda=0$. When writing $S(0)$ we will implicitly assume that only the coefficients $S_{\mu \nu}$ with nonzero $\mu$ and $\nu$ are considered (see also remark 5.5).

In the next subsection we construct solutions to the problems (4.2, 4.3) since they give the functions $G_{\nu}(\cdot ; 0)$ from which the coefficients $S_{\mu \nu}$ can be computed (for nonzero $\mu$ and $\nu$ ).

4.3. Special solutions and an explicit expression for $S(0)$. Choose a canonical basis of cycles, $\left\{a_{\alpha}, b_{\alpha}\right\}$ on the Riemann surface $X$ and let $\left\{v_{\alpha}\right\}_{\alpha=1, \ldots, g}$ be the corresponding basis of holomorphic normalized differentials. Let $\mathbb{B}$ be the matrix of $b$-periods of $X$.

We have the following proposition.

Proposition 4.6. Fixing $P$ a conical point and $k \in \mathbb{N}$, there exist $\Omega_{k}$ and $\Sigma_{k}$ such that

(1) $\Omega_{k}$ and $\Sigma_{k}$ are meromorphic differentials of the second kind on $X$ with only one pole of order $k+1$ at $P$.

(2) In the distinguished local parameter near P, they satisfy

$$
\left\{\begin{array}{l}
\Omega_{k}(\zeta)=-\frac{k}{\zeta^{k+1}} d \zeta+O(1) \\
\Sigma_{k}(\zeta)=-\frac{i k}{\zeta^{k+1}} d \zeta+O(1) .
\end{array}\right.
$$

(3) All the $a$ and $b$-periods of $\Omega_{k}(P, \cdot)$ and $\Sigma_{k}(P, \cdot)$ are purely imaginary.

Proof. Let $\omega(\cdot, \cdot)$ be the canonical meromorphic bidifferential on the Riemann surface $X$ (see [9], p. 3 ), for which the following asymptotic expansion holds

$$
\frac{\omega\left(\zeta\left(Q_{1}\right), \zeta\left(Q_{2}\right)\right)}{d \zeta\left(Q_{1}\right) d \zeta\left(Q_{2}\right)}=\frac{1}{\left(\zeta\left(Q_{1}\right)-\zeta\left(Q_{2}\right)\right)^{2}}+\frac{1}{6} S_{B}\left(\zeta\left(Q_{2}\right)\right)+o(1)
$$

as $Q_{1} \rightarrow Q_{2}$, where $S_{B}$ is the Bergman projective connection. Moreover, $\omega$ is normalized in such a way that

$$
\left\{\begin{array}{l}
\left.\oint_{a_{\alpha}} \frac{\omega(\cdot, \zeta)}{d \zeta}\right|_{\zeta=0}=0 \\
\left.\oint_{b_{\alpha}} \frac{\omega(\cdot, \zeta)}{d \zeta}\right|_{\zeta=0}=\left.2 \pi i \frac{v_{\alpha}(\zeta)}{d \zeta}\right|_{\zeta=0},
\end{array}\right.
$$

for $\alpha=1, \cdots, g$.

Let $\left(c_{\alpha}\right)_{\alpha=1 \cdots g}$ be coefficients to be chosen later and consider the meromorphic differential

$$
-\left.\frac{\omega(\cdot, \zeta)}{d \zeta}\right|_{\zeta=0}+\sum_{\alpha=1}^{g} c_{\alpha} v_{\alpha} .
$$


We want to choose $c_{\alpha}$ in (4.6) so that all the $a$ - and $b$-periods of this differential are purely imaginary. The vanishing of the real parts of all $a$-periods implies that all the constants $c_{\alpha}$ are purely imaginary. The vanishing of the real part of the period over the cycle $b_{\beta}$ then gives :

$$
\operatorname{Re}\left(\oint_{b_{\beta}} \sum c_{\alpha} v_{\alpha}\right)=\operatorname{Re}\left(\left.\oint_{b_{\beta}} \frac{\omega(\cdot, \zeta)}{d \zeta}\right|_{\zeta=0}\right) .
$$

Using the fact that the $c_{\alpha}$ are known to be purely imaginary and the normalization of $\omega$ recalled in (4.5) we obtain the following system of equations :

$$
\sum_{\alpha=1}^{g}[\operatorname{Im} \mathbb{B}]_{\beta \alpha} c_{\alpha}=2 \pi i \operatorname{Im}\left(\left.\frac{v_{\beta}}{d \zeta}\right|_{\zeta=0}\right)
$$

Since $\operatorname{Im}(\mathbb{B})$ is invertible, this uniquely determines $c_{\alpha}$.

In order to get $\Sigma_{1}$ we apply the same method searching coefficients $c_{\alpha}$ so that the meromorphic differential

$$
-i \frac{\omega(\cdot, \zeta)}{d \zeta}+\sum_{\alpha=1}^{g} c_{\alpha} v_{\alpha}
$$

has purely imaginary periods. The system of equations we obtain is similar to (4.7) except that $\operatorname{Im}\left(\left.\frac{v_{\beta}}{d \zeta}\right|_{\zeta=0}\right)$ is replaced by $\operatorname{Re}\left(\left.\frac{v_{\beta}}{d \zeta}\right|_{\zeta=0}\right)$. It still has a solution using the same invertibility of $\operatorname{Im}(\mathbb{B})$.

To get $\Omega_{k}$ and $\Sigma_{k}$ with an arbitrary $k \geq 1$ we repeat the same construction taking the first term in (4.6) to be

$$
\left.\frac{(-1)^{k}}{(k-1) !}\left[\frac{d}{d \zeta}\right]^{k-1} \frac{\omega(\cdot, \zeta)}{d \zeta}\right|_{\zeta=0} .
$$

We will obtain an equation similar to (4.7) so that eventually, the existence result thus follows from the existence of $\omega$ and the fact that the matrix $\operatorname{Im}(\mathbb{B})$ is invertible.

This proposition gives the following corollary.

Corollary 4.7. Let $\Omega_{k}$ and $\Sigma_{k}$ be defined by the preceding proposition, then the following formula defines a function $f_{k}$ which is harmonic in $X \backslash\{P\}$ :

$$
f_{k}(Q)=\operatorname{Re}\left\{\int_{P_{0}}^{Q} \Omega_{k}\right\}-i R e\left\{\int_{P_{0}}^{Q} \Sigma_{k}\right\}
$$

Moreover, in the distinguished local parameter near $P, f_{k}$ admits the following asymptotic behavior:

$$
f_{k}(\zeta)=\frac{1}{\zeta^{k}}+O(1)
$$

Proof. Since all the $a$ - and $b$ - periods of $\Omega$ and $\Sigma$ are purely imaginary, $f_{k}$ is indeed well-defined on $X$. The remaining statements follow from the construction.

By considering $C_{\nu} f_{k}$ or $C_{\nu} \overline{f_{k}}$ we obtain the functions $G_{\nu}(\cdot ; 0)$ up to an additive constant. This additive constant is harmless when computing the matrix elements $S_{\mu \nu}(0)$. 
4.3.1. Examples.

(1) A conical point of angle $2 \pi<\beta \leq 4 \pi$ on a Euclidean surface of genus $\geq 1$. In this case one has $n=1$.

The proposition 4.6 combined with the asymptotics of $\omega$ yield

$$
\begin{aligned}
\int_{P_{0}}^{\zeta} \Omega_{1}(P, \cdot)=\frac{1}{\zeta}+c_{0}+ & {\left[-\left.\frac{1}{6} S_{B}(\zeta)\right|_{\zeta=0}+\left.2 \pi i \sum_{\alpha=1, \beta=1}^{g}\left((\operatorname{Im~BB})^{-1}\right)_{\alpha \beta} \operatorname{Im}\left\{\left.\frac{v_{\beta}(\zeta)}{d \zeta}\right|_{\zeta=0}\right\} \frac{v_{\alpha}(\zeta)}{d \zeta}\right|_{\zeta=0}\right] } \\
& +O\left(\zeta^{2}\right)
\end{aligned}
$$

with some constant $c_{0}$, and

$$
\begin{aligned}
\int_{P_{0}}^{\zeta} \Sigma_{1}(P, \cdot)=\frac{i}{\zeta}+d_{0}+ & {\left[-\left.\frac{i}{6} S_{B}(\zeta)\right|_{\zeta=0}+\left.2 \pi i \sum_{\alpha=1, \beta=1}^{g}\left((\operatorname{Im~BB})^{-1}\right)_{\alpha \beta} \operatorname{Re}\left\{\left.\frac{v_{\beta}(\zeta)}{d \zeta}\right|_{\zeta=0}\right\} \frac{v_{\alpha}(\zeta)}{d \zeta}\right|_{\zeta=0}\right] } \\
& +O\left(\zeta^{2}\right)
\end{aligned}
$$

with some constant $d_{0}$.

Denoting the expressions in square brackets in (4.9) and (4.10) by $A$ and $B$ respectively, one gets the asymptotics

$$
f_{1}(\zeta, \bar{\zeta})=\frac{1}{\zeta}+\text { const }+\frac{A-i B}{2} \zeta+\frac{\bar{A}-i \bar{B}}{2} \bar{\zeta}+O\left(|\zeta|^{2}\right)
$$

and, therefore,

$$
S_{p}(0)=\left(\begin{array}{crr}
* & * & * \\
* & \frac{A-i B}{2} & \frac{\bar{A}-i \bar{B}}{2} \\
* & \frac{A+i B}{2} & \frac{\bar{A}+i \bar{B}}{2}
\end{array}\right),
$$

where the index $p$ means that we have written down only the coefficients of $S(0)$ that corresponds to indices $\nu \in N_{p}$

(2) A Euclidean sphere with one $4 \pi$ singularity and six $\pi$ singularities. Consider the surface of example 2.1 i.e. the Riemann sphere with metric

$$
\frac{|z|^{2}|d z|^{2}}{\prod_{k=1}^{6}\left|z-z_{k}\right|}
$$

We consider the part of the $S$-matrix with non-zero indices $\mu$ and $\nu$. We thus only have to consider the asymptotic behavior near 0 and compute the coefficients $S_{\frac{1}{2} \frac{1}{2}}, S_{-\frac{1}{2}-\frac{1}{2}}, S_{-\frac{1}{2} \frac{1}{2}}$ and $S_{\frac{1}{2}-\frac{1}{2}}$.

${ }^{2}$ The distinguished local parameter $\zeta$ in a vicinity of the conical point $z=0$ is given by

$$
\zeta(z)=\left(\int_{0}^{z} \frac{w d w}{\sqrt{\prod_{k=1}^{6}\left(w-z_{k}\right)}}\right)^{1 / 2} .
$$

The special solution $f_{1}$ is now not only harmonic but even holomorphic in $\mathbb{C} P^{1} \backslash 0$ and is nothing but the function $A / z$ with some constant $A$. 
One has

$$
\frac{A}{z}=\frac{1}{\zeta}+\text { const }+S_{\frac{1}{2} \frac{1}{2}}(0) \zeta+O\left(\zeta^{2}\right),
$$

Therefore, $A=\left.\frac{d z}{d \zeta}\right|_{\zeta=0}$ and a simple calculation shows that

$$
S_{\frac{1}{2} \frac{1}{2}}(0)=-\left.\frac{1}{6} \frac{z^{\prime \prime \prime}(\zeta) z^{\prime}(\zeta)-\frac{3}{2}\left(z^{\prime \prime}(\zeta)\right)^{2}}{\left(z^{\prime}(\zeta)^{2}\right.}\right|_{\zeta=0}=-\left.\frac{1}{6}\{z, \zeta\}\right|_{\zeta=0}
$$

where $\{z, \zeta\}$ is the Schwarzian derivative. One has also $S_{-\frac{1}{2}-\frac{1}{2}}(0)=\overline{S_{\frac{1}{2} \frac{1}{2}}(0)}$ and $S_{\frac{1}{2}-\frac{1}{2}}(0)=$ $S_{-\frac{1}{2} \frac{1}{2}}(0)=0$.

In the very symmetric case where the $z_{k}$ form a regular hexagon, the computation yields that $z=c \cdot \zeta\left(1+O\left(\zeta^{6}\right)\right)$ so that $S_{\frac{1}{2} \frac{1}{2}}$ and $S_{-\frac{1}{2}-\frac{1}{2}}$ also vanish.

4.4. $S$-matrix as a function on the moduli space of holomorphic differentials: variational formulas. Let $(X, \omega) \in H_{g}\left(k_{1}, \ldots, k_{M}\right)$ and let $S(\lambda)$ be the $S$-matrix corresponding to a conical point of the translation surface $\left(X,|\omega|^{2}\right)$ (i. e. one of the zeros of the holomorphic one-form $\omega$ ). Here we derive the variational formulas for $S(\lambda)$ with respect to Kontsevich-Zorich homological coordinates on $H_{g}\left(k_{1}, \ldots, k_{M}\right)$.

Proposition 4.8. Let $z(p)=\int^{p} \omega$. Introduce the following (closed) (1-1)-form on $X_{0}$ :

$$
\Theta_{\mu \nu}=\left[G_{\mu}(z ; \lambda)\right]_{z \bar{z}} G_{\nu}(z ; \lambda) d \bar{z}+\left[G_{\mu}(z ; \lambda)\right]_{z}\left[G_{\nu}(z ; \lambda)\right]_{z} d z,
$$

Then the variational formulas hold:

$$
\begin{gathered}
\frac{\partial S_{\mu \nu}(\lambda)}{\partial A_{\alpha}}=2 i \oint_{b_{\alpha}} \Theta_{\mu \nu} ; \quad \alpha=1, \ldots, g, \\
\frac{\partial S_{\mu \nu}(\lambda)}{\partial B_{\alpha}}=-2 i \oint_{a_{\alpha}} \Theta_{\mu \nu} ; \quad \alpha=1, \ldots, g, \\
\frac{\partial S_{\mu \nu}(\lambda)}{\partial z_{k}}=2 i \oint_{p_{k}} \Theta_{\mu \nu} ; \quad k=2, \ldots, M,
\end{gathered}
$$

where the integrals in (4.14) are taken over some small contours encircling conical points $p_{k}$.

Proof. The method of proof follows closely [19]. We will prove only the variational formulas with respect to coordinates $A_{\alpha}$ since the other formulas can be established similarly.

According to [19] (Proposition 2, p. 84) one has

$$
\partial_{A_{\alpha}} R(x, y ; \lambda)=2 i \oint_{b_{\alpha}} R(x, z ; \lambda) R_{z \bar{z}}(y, z ; \lambda) d \bar{z}+R_{z}(x, z ; \lambda) R_{z}(y, z ; \lambda) d z .
$$

(Here $R(x, y ; \lambda)$ stands for the resolvent kernel of the Friedrichs extension; one has $R_{z \bar{z}}(x, z ; \lambda)=$ $\frac{\lambda}{4} R(x, z ; \lambda)$. ) On the other hand, by definition of $g_{\nu}$ we have

$$
g_{\nu}(x ; \lambda)=-\iint_{X}\left[R(x, y ; \lambda)(\Delta-\lambda) F_{\nu}(y)\right] d y ;
$$

and Lemma 7 on page 88 of [19] reads as

$$
\partial_{A_{\alpha}} \iint_{X} \Phi(x, \bar{x} ; \text { moduli }) d x=\iint_{X} \partial_{A_{\alpha}} \Phi(x, \bar{x}, \text { moduli }) d x+\frac{i}{2} \oint_{b_{\alpha}} \Phi(x, \bar{x}, \text { moduli }) d \bar{x} .
$$


The cycle $b_{\alpha}$ does not intersect the support of $F_{\nu}$ and the terms $F_{\nu}$ and $(\Delta-\lambda) F_{\nu}$ are moduli independent, therefore,

$$
\partial_{A_{\alpha}} G_{\nu}(x ; \lambda)=\partial_{A_{\alpha}}\left(F_{\nu}+g_{\nu}\right)=\partial_{A_{\alpha}} g_{\nu}(x ; \lambda) .
$$

Using (4.16) and (4.17), we obtain

$$
\begin{aligned}
\partial_{A_{\alpha}} G_{\nu}(x ; \lambda)= & 2 i \iint_{X} d y\left[(\Delta-\lambda) F_{\nu}(y)\right] \oint_{b_{\alpha}}\left\{R(x, z ; \lambda) R_{z \bar{z}}(y, z ; \lambda) d \bar{z}+R_{z}(x, z ; \lambda) R_{z}(y, z ; \lambda) d z\right\} \\
= & 2 i \oint_{b_{\alpha}} R(x, z ; \lambda)\left[\iint_{X} \frac{\lambda}{4} R(y, z ; \lambda)(\Delta-\lambda) F_{\nu}(y) d y\right] d \bar{z} \\
& +2 i \oint_{b_{\alpha}} R_{z}(x, z ; \lambda)\left[\iint_{X} R_{z}(y, z ; \lambda)(\Delta-\lambda) F_{\nu}(y) d y\right] d z \\
= & 2 i \oint_{b_{\alpha}} R_{z \bar{z}}(x, z ; \lambda) g_{\nu}(z ; \lambda) d \bar{z}+R_{z}(x, z ; \lambda)\left[g_{\nu}(z ; \lambda)\right]_{z} d z \\
= & 2 i \oint_{b_{\alpha}} R_{z \bar{z}}(x, z ; \lambda) G_{\nu}(z ; \lambda) d \bar{z}+R_{z}(x, z ; \lambda)\left[G_{\nu}(z ; \lambda)\right]_{z} d z
\end{aligned}
$$

We finally obtain

$$
\partial_{A_{\alpha}} g_{\nu}(\lambda, x)=2 i \oint_{b_{\alpha}} R_{z \bar{z}}(x, z ; \lambda) G_{\nu}(z ; \lambda) d \bar{z}+R_{z}(x, z ; \lambda)\left[G_{\nu}(z ; \lambda)\right]_{z} d z .
$$

Using proposition 3.13 and equation (3.10) to identify the behavior near the conical points of the different terms we obtain

$$
\partial_{A_{\alpha}} S_{\mu \nu}=2 i \oint_{b_{\alpha}}\left[a_{\mu}^{+}(z ; \lambda)\right]_{z \bar{z}} G_{\nu}(z ; \lambda) d \bar{z}+\left[a_{\mu}^{+}(z ; \lambda)\right]_{z}\left[G_{\nu}(z ; \lambda)\right]_{z} d z
$$

Using proposition 3.13 , this gives the result.

\section{KREIN'S FORMUla AND RELATIVE DETERMinANTS}

There are several ways of defining determinants of operators acting on an infinite dimensional space. We recall the following two basic constructions : first a perturbative determinant when the operator is a trace-class perturbation of the identity, and second zeta-regularization which is used in particular for Laplacian-like operators.

Both these approaches can also be used to define relative determinants when comparing two operators $H_{0}$ and $H_{1}$ in which one is thought to be a perturbation of the other. Krein's formula is a classical tool in this setting and usually applies when the difference $f\left(H_{1}\right)-f\left(H_{0}\right)$ is trace-class for some simple function $f$. In that case it is possible to define a relative perturbative determinant (see [29]). This approach applies well to the case when $H_{0}$ and $H_{1}$ are different self-adjoint extensions of a symmetric operator that has finite deficiency indices. Indeed, in that case the difference of the resolvents is a finite-rank operator and, moreover, the perturbative determinant is actually the determinant of a finite dimensional matrix.

We will thus adapt these techniques to our setting. The method is clearly identified in the literature (see [29] and also [5]) and the main task here consists in identifying the perturbative determinant in terms of the boundary condition and the $S$-matrix.

Once this is done, we will use this determinant to define a zeta-regularization and compare the determinants that are obtained this way.

Remark 5.1. We insist here that we will actually use the perturbative determinant to show that zetaregularization is possible and then to compare the two definitions of determinants. In particular, all 
the issues that are relative to zeta-regularization may be expressed using the perturbative determinant (when the latter can be defined).

5.1. Krein's formula and perturbative determinant. One convenient way of parametrizing the self-adjoint extensions of $\Delta$ is by using two matrices $P$ and $Q$ in the following way (see [21]).

We first construct two vectors $A^{ \pm}$that collect the coefficients $a_{\nu}^{ \pm}$. We organize these coefficients so that the first $n_{p_{1}}$ entries correspond to the first conical point $p_{1}$ then we put the data corresponding to the second conical points and so on.

A lagrangian subspace $L$ in $\operatorname{dom}\left(\Delta^{*}\right) / \operatorname{dom}(\Delta)$ can be parametrized by a system of linear equations of the following form :

$$
P A^{-}+Q A^{+}=0,
$$

where $P$ and $Q$ are square matrices satisfying $\operatorname{rank}(P, Q)$ is maximal and $P^{*} Q$ is self-adjoint. We fix two such matrices and denote by $\Delta_{L}$ the corresponding self-adjoint extensions.

It is possible to find a basis in which the $n \times 2 n$ matrix $(P Q)$ has the following block-decomposition $(21]):$

$$
\left(\begin{array}{cccc}
P_{2} & P_{3} & Q_{1} & 0 \\
0 & P_{1} & 0 & 0
\end{array}\right)
$$

in which $P_{1}$ and $Q_{1}$ are invertible and $L:=Q_{1}^{-1} P_{2}$ is self-adjoint.

Definition 5.2. We will call an extension $\Delta_{L}$ regular if functions in $\operatorname{dom}\left(\Delta_{L}\right)$ are not allowed to have logarithmic singularities. Equivalently, $\Delta_{L}$ is regular if and only if for any $u \in \operatorname{dom}\left(\Delta_{L}\right)$, and any conical point $p$, the coefficient $a_{p, 0}^{-}$of $u$ vanishes.

The following observation (based on the classical Krein formula) is the key technical result of the present paper.

Proposition 5.3. For any $\lambda \in \mathbb{C} \backslash\left(\operatorname{spec}\left(\Delta_{\mathrm{F}}\right) \cup \operatorname{spec}\left(\Delta_{\mathrm{L}}\right)\right)$ the following identity holds :

$$
\operatorname{Tr}\left(\left(\Delta_{L}-\lambda\right)^{-1}-\left(\Delta_{F}-\lambda\right)^{-1}\right)=-\operatorname{Tr}\left((P+Q S(\lambda))^{-1} Q \dot{S}(\lambda)\right),
$$

where the dot indicates derivation with respect to $\lambda$.

Proof. Let $\lambda$ be in the union of the resolvent sets of $\Delta_{F}$ and $\Delta_{L}$, and let $f$ be in $L^{2}$. We search a matrix $X=\left[x_{\mu \nu}\right]$ such that we have the following Krein formula (see, e. g., [4] or [1], Theorem A.3)

$$
\left(\Delta_{L}-\lambda\right)^{-1} f=\left(\Delta_{F}-\lambda\right)^{-1}(f)+\sum_{\mu, \nu} x_{\mu \nu} G_{\mu}(\cdot ; \lambda) \Lambda_{\nu}\left[\left(\Delta_{F}-\lambda\right)^{-1}(f)\right] .
$$

We denote by $u=\left(\Delta_{F}-\lambda\right)^{-1}(f)$ and we compute the vectors $A^{ \pm}$of the right-hand side

$$
\begin{gathered}
a_{\mu}^{-}=\sum_{\nu} x_{\mu \nu} \Lambda_{\nu}(u), \\
a_{\mu^{\prime}}^{+}=\Lambda_{\mu^{\prime}}(u)+\sum_{\mu, \nu} x_{\mu \nu}[S(\lambda)]_{\mu^{\prime} \mu} \Lambda_{\nu}(u) .
\end{gathered}
$$

Denoting by $\Lambda$ the vector $\Lambda_{\nu}(u)$ we thus have

$$
A^{-}=X \Lambda, A^{+}=(I+S(\lambda) X) \Lambda .
$$

Plugging into the self-adjoint condition we obtain that the following relation is satisfied.

$$
[P X+Q(I+S(\lambda) X)] \cdot \Lambda=0
$$


Using the block decomposition (5.1), we see that

$$
P+Q S(\lambda)=\left(\begin{array}{cc}
P_{2}+Q_{1} S(\lambda) & * \\
0 & P_{1}
\end{array}\right)
$$

Since $\lambda$ is in both resolvent sets, $\Lambda$ is arbitrary and the preceding system always has a solution. We obtain that $\left(P_{2}+Q_{1} S(\lambda)\right)$ must be invertible and hence $P+Q S(\lambda)$. Finally, we obtain

$$
X=-(P+Q S(\lambda))^{-1} Q .
$$

Denoting by $\Pi_{\mu \nu}(\lambda)$ the (rank one) operator defined from $H^{2}$ into $L^{2}$ by

$$
\Pi_{\mu \nu}(\lambda)(u)=G_{\mu}(\cdot ; \lambda) \Lambda_{\nu}(u)
$$

equation (5.2) may be rewritten :

$$
\left(\Delta_{L}-\lambda\right)^{-1}-\left(\Delta_{F}-\lambda\right)^{-1}=\sum_{\mu, \nu} x_{\mu \nu} \Pi_{\mu \nu}(\lambda) \circ\left(\Delta_{F}-\lambda\right)^{-1}
$$

Observe that the right-hand side is finite rank so that we can trace this equation and obtain

$$
\left.\operatorname{Tr}\left(\left(\Delta_{L}-\lambda\right)^{-1}-\left(\Delta_{F}-\lambda\right)^{-1}\right)\right)=\sum_{\mu, \nu} x_{\mu \nu} \operatorname{Tr}\left(\Pi_{\mu \nu}(\lambda) \circ\left(\Delta_{F}-\lambda\right)^{-1}\right) .
$$

Using lemma 5.4 below and lemma 3.12 we obtain

$$
\begin{aligned}
\left.\operatorname{Tr}\left(\left(\Delta_{L}-\lambda\right)^{-1}-\left(\Delta_{F}-\lambda\right)^{-1}\right)\right) & =\sum_{\mu, \nu} x_{\mu \nu}\left\langle G_{\mu}(\cdot ; \lambda), G_{\nu}(\cdot ; \bar{\lambda})\right\rangle \\
& =\sum_{\mu \nu} x_{\mu \nu}[\dot{S}(\lambda)]_{\nu \mu} \\
& =-\operatorname{Tr}\left((P+Q S(\lambda))^{-1} Q \dot{S}(\lambda)\right)
\end{aligned}
$$

It remains to prove the following lemma.

Lemma 5.4. The trace of the rank one operator $\Pi_{\mu \nu}(\lambda) \circ\left(\Delta_{F}-\lambda\right)^{-1}$ is given by

$$
\operatorname{Tr}\left(\Pi_{\mu \nu}(\lambda) \circ\left(\Delta_{F}-\lambda\right)^{-1}\right)=\left\langle G_{\mu}(\cdot ; \lambda), G_{\nu}(\cdot ; \bar{\lambda})\right\rangle
$$

Proof. Let $e_{n}$ be an orthonormal basis of $L^{2}$ then

$$
\begin{aligned}
\left\langle\Pi_{\mu \nu}(\lambda) \circ\left(\Delta_{F}-\lambda\right)^{-1} e_{n}, e_{n}\right\rangle & =\left\langle G_{\mu}(\cdot ; \lambda), e_{n}\right\rangle \cdot \Lambda_{\nu}\left(\left(\Delta_{F}-\lambda\right)^{-1} e_{n}\right) \\
& =\left\langle G_{\mu}(\cdot ; \lambda), e_{n}\right\rangle \cdot\left\langle e_{n}, G_{\nu}(\cdot ; \bar{\lambda})\right\rangle
\end{aligned}
$$

Summing over $n$ and using Parseval's identity gives the lemma.

We may now define $D$ on the union of the resolvent sets of $\Delta_{F}$ and $\Delta_{L}$ by

$$
D(\lambda)=\operatorname{det}(P+Q S(\lambda)) .
$$

Remark 5.5. When the extension is regular the matrix $P+Q S(\lambda)$ doesn't involve the coefficients $S_{\mu \nu}$ whenever $\mu$ or $\nu$ is 0 (because these are multiplied by a zero entry of $Q$ ). Hence the matrix $P+Q S(0)$ makes perfect sense and can be computed using the results of section 4.2. 
The preceding proposition gives

$$
\operatorname{Tr}\left(\left(\Delta_{L}-\lambda\right)^{-1}-\left(\Delta_{F}-\lambda\right)^{-1}\right)=-\frac{D^{\prime}(\lambda)}{D(\lambda)}
$$

This implies that $\frac{D^{\prime}}{D}$ extends to a meromorphic function with poles that correspond to eigenvalues of $\Delta_{L}$ and $\Delta_{F}$ and with residues $\operatorname{dim}\left(\operatorname{ker}\left(\Delta_{L}-\lambda\right)\right)-\operatorname{dim}\left(\operatorname{ker}\left(\Delta_{F}-\lambda\right)\right)$.

Since $\frac{D^{\prime}}{D}$ is the logarithmic derivative of $D$, it is convenient to give a name to $\ln (D)$. We thus denote by $\Omega \subset \mathbb{C}$ the set obtained by removing a downward vertical cut starting at each eigenvalue of $\Delta_{F}$ and $\Delta_{L}$ i.e.

$$
\Omega=\mathbb{C} \backslash\left\{\lambda-i t, \lambda \in \operatorname{spec}\left(\Delta_{F}\right) \cup \operatorname{spec}\left(\Delta_{L}\right), t \in(-\infty, 0]\right\},
$$

and, on $\Omega$, we define the function $\tilde{\xi}$ by $\tilde{\xi}(\lambda):=-\frac{1}{2 i \pi} \ln (\operatorname{det}(P+Q S(\lambda)))$.

Observe that on $\Omega$ we have, by definition,

$$
D(\lambda)=\exp (-2 i \pi \tilde{\xi}(\lambda))
$$

The function $\tilde{\xi}$ is intimately related to the spectral shift function $\xi$ (see [29, 13]). Although the latter is usually used in settings with continuous spectrum, it is possible to define it even when $H_{0}$ and $H_{1}$ have pure point spectrum. In the latter case, it follows from the definitions that $\xi$ is the step-function : $\xi(t):=N_{1}(t)-N_{0}(t)$ where $N_{i}$ is the counting function associated with $H_{i}$.

It follows from our definition of $\tilde{\xi}$ that the function $\xi$ defined on $\mathbb{R}$ by

$$
\xi(t):=-\frac{1}{2 \pi} \operatorname{Arg}(D(t))=\operatorname{Re} \tilde{\xi}(t)
$$

is a step function with jumps located at the eigenvalues of $\Delta_{F}$ and $\Delta_{L}$. Moreover the jumps are exactly the differences $\operatorname{dim}\left(\operatorname{ker}\left(\Delta_{L}-\lambda\right)\right)-\operatorname{dim}\left(\operatorname{ker}\left(\Delta_{F}-\lambda\right)\right)$. We thus obtain the spectral shift function of $\Delta_{F}$ and $\Delta_{L}$. (compare with [29] Thm 1 p. 272).

In our setting Birman-Krein formula would be (5.5) (compare with 29] p.272) and would follow, in our case, from our definitions. In the next subsection we will prove that, using $D$, one may define a determinant of $\Delta_{L}$ via zeta-regularization and then establish the relation :

$$
\operatorname{det}_{\zeta}\left(\Delta_{L}-\lambda\right)=C_{0} \cdot D(\lambda) \operatorname{det}_{\zeta}\left(\Delta_{F}-\lambda\right),
$$

in which $C_{0}$ is some constant that we will also determine.

In particular, we will now prove that $D$ allows us to recover the 'exotic' features of the zeta function associated with $\Delta_{L}$. This unusual behavior has been extensively studied in [17] in a setting very close to ours and in 12 in greater generality. Our main contribution here is the interpretation of $D$ using $S$-matrix that, in some sense, gives a geometrical interpretation to the 'secular equation' method of [17.

5.2. Comparing determinants. The procedure here is not as straightforward as usual because of unusual behavior of the zeta function near $s=0$. In particular, $\zeta\left(s, \Delta_{L}\right)$ will admit a analytic continuation that is regular at 0 only if $L$ is regular (though with possible unusual poles). This unusual behavior as we just mentioned has been extensively studied in literature (see [12, 17, 22]); from our point of view, it is linked with the asymptotic behavior of $D(\lambda)$ for large negative $\lambda$. We thus begin by deriving this asymptotic expansion. 
5.2.1. $D(\lambda)$ for large negative $\lambda$. The analysis that follows is closely related to the one performed in [17. This is not surprising since the asymptotic regime $\lambda$ goes to $-\infty$ is local. In particular, the function $D(-|\lambda|):=\operatorname{det}(P+Q S(-|\lambda|))$ on a cone has to be compared to the function $F(i \sqrt{|\lambda|})$ in 17.

We first use prop. 4.2 and consider all possible sums of the exponents $\nu_{i}$ that appear in this proposition. This gives us a collection of numbers that we order and denote by $\alpha_{0}>\alpha_{1}>\cdots>$ $\alpha_{k}>\cdots$. Expanding now the determinant, and ordering the terms, we get

$$
D(-|\lambda|)=\sum_{\text {finite }} a_{k l}|\lambda|^{\alpha_{k}}(\ln |\lambda|)^{l}+O\left(|\lambda|^{-\infty}\right) .
$$

By definition, there are no logarithm in the expansion corresponding to a regular self-adjoint extension, therefore, in that case, the expansion reads :

$$
D(-|\lambda|)=\sum_{\text {finite }} a_{k}|\lambda|^{\alpha_{k}}+O\left(|\lambda|^{-\infty}\right) .
$$

We set $l_{k}$ the largest integer $l$ such that $|\lambda|^{\alpha_{k}}(\ln |\lambda|)^{l}$ appears in the expansion and we set $\beta_{k}=\alpha_{0}-\alpha_{k}$ we have

$$
D(-|\lambda|)=a_{0 l_{0}}|\lambda|^{\alpha_{0}}(\ln |\lambda|)^{l_{0}}\left[1+\sum_{l \geq 1} a_{0 l}(\ln |\lambda|)^{-l}+\sum_{\beta_{k}>0} \sum_{-l_{k}}^{l_{0}} a_{k l}|\lambda|^{-\beta_{k}}(\ln |\lambda|)^{l}+O\left(|\lambda|^{-\infty}\right)\right]
$$

We denote by $F(\lambda)=\left[1+\sum_{l \geq 1} a_{0 l}(\ln |\lambda|)^{-l}+\sum_{\beta_{k}>0} \sum_{-l_{k}}^{l_{k}} a_{k l}|\lambda|^{-\beta_{k}}(\ln |\lambda|)^{l}+O\left(|\lambda|^{-\infty}\right)\right]$

Taking the logarithmic derivative, we obtain

$$
-\frac{D^{\prime}(-|\lambda|)}{D(-|\lambda|)}=2 i \pi \tilde{\xi}^{\prime}(-|\lambda|)=\frac{\alpha_{0}}{|\lambda|}+\frac{l_{0}}{|\lambda| \ln |\lambda|}+\frac{F^{\prime}(\lambda)}{F(\lambda)} .
$$

By inspection we find

$$
\frac{F^{\prime}(\lambda)}{F(\lambda)}=\left\{\begin{array}{lr}
O\left(|\lambda|^{-\beta_{1}-1}\right) & \text { regular case } \\
O\left(|\lambda|^{-1}(\ln |\lambda|)^{-2}\right) & \text { otherwise. }
\end{array}\right.
$$

Lemma 5.6. (1) In the regular case, there exist three positive numbers $\alpha_{0}, \beta_{1}$ and $M$ such that the estimate

$$
\left|2 i \pi \tilde{\xi}^{\prime}(-|\lambda|)-\frac{\alpha_{0}}{|\lambda|}\right| \leq M|\lambda|^{-\beta_{1}-1}
$$

holds for $\lambda$ large enough.

(2) In the other cases, there exist two positive real numbers $\alpha_{0}$ and $\beta_{1}$, a non-negative integer $l_{0}$ and a constant $M$ such that the estimate

$$
\left|2 i \pi \tilde{\xi}^{\prime}(-|\lambda|)-\frac{\alpha_{0}}{|\lambda|}-\frac{l_{0}}{|\lambda| \ln |\lambda|}\right| \leq M \cdot|\lambda|^{-1}(\ln |\lambda|)^{-2}
$$

holds for $|\lambda|$ large enough.

In the regular case, for any $C>0$, define $h_{C}(s)$ for $\operatorname{Re}(s)$ large enough by

$$
h_{C}(s)=2 i \pi \int_{C}^{\infty} \lambda^{-s} \tilde{\xi}^{\prime}(-|\lambda|) d \lambda-\frac{\alpha_{0}}{s} \exp (-s \ln (C)) .
$$


The estimates of the previous lemma imply the following corollary. We restrict to the regular case although similar statements are valid in the non-regular case (with extra logarithmic singularities -see [17]).

Proposition 5.7. For a regular extension, the function $h_{C}$ extends to a holomorphic function on $\left\{\operatorname{Re}(s) \geq-\beta_{1}\right\}$.

Proof. We have

$$
\begin{aligned}
\int_{C}^{\infty} \lambda^{-s} 2 i \pi \tilde{\xi}^{\prime}(-|\lambda|) d \lambda= & \int_{C}^{\infty} \lambda^{-s}\left[2 i \pi \tilde{\xi}^{\prime}(-|\lambda|)-\frac{\alpha_{0}}{\lambda}\right] d \lambda \\
& +\int_{C}^{\infty} \lambda^{-s} \frac{\alpha_{0}}{\lambda} d \lambda
\end{aligned}
$$

The second integral on the right-hand side is computed directly :

$$
\int_{C}^{\infty} \lambda^{-s} \frac{\alpha_{0}}{\lambda} d \lambda=\frac{\alpha_{0}}{s} \exp (-s \ln C)
$$

so that $h_{C}$ actually represents the first integral. Lemma 5.6 then gives that $h_{C}$ extends to a holomorphic function on $\operatorname{Re} s>-\beta_{1}$.

5.3. Zeta-regularization. For any $A$ and any $C$ that is large enough, for any $\tilde{\lambda} \in \Omega$ such that $\operatorname{Re}(\tilde{\lambda})>A$ we choose a cut $c_{\tilde{\lambda}} \subset \Omega$ that starts from $-\infty+i 0$ and that ends at $\tilde{\lambda}$. We may choose it in such a way that it begins with the interval $(-\infty,-C]$.

For any $\tilde{\lambda}$ and any $s \in \mathbb{C}$, the function $\lambda \mapsto(\lambda-\tilde{\lambda})^{-s}$, which is well defined when $\lambda-\tilde{\lambda}$ is a positive real number, extends to a holomorphic function on the complement of the cut $c_{\tilde{\lambda}}$. Moreover, when $\lambda$ goes to the cut $c_{\tilde{\lambda}}$ from above or from below, we have the following jump condition

$$
\lim _{\lambda \downarrow c_{\tilde{\lambda}}} \exp (-i \pi s)(\lambda-\tilde{\lambda})^{-s}=\lim _{\lambda \uparrow c_{\tilde{\lambda}}} \exp (i \pi s)(\lambda-\tilde{\lambda})^{-s} .
$$

For $\lambda$ on $c_{\tilde{\lambda}}$, we define $(\lambda-\tilde{\lambda})_{0}^{-s}$ to be this common limit.

Let $A^{+}$be any number greater than $A$ that is neither an eigenvalue of $\Delta_{F}$ nor of $\Delta_{L}$. Define a contour $\gamma$ that avoids $c_{\tilde{\lambda}}$ and that consists in one part that encloses the half-line $\left\{x \geq A^{+}\right\}$and then small circles that enclose the eigenvalues of $\Delta_{L}$ and $\Delta_{F}$ that are smaller than $A^{+}$.

For $\operatorname{Re}(s)>1$ we have

$$
\begin{aligned}
\zeta\left(s, \Delta_{L}-\tilde{\lambda}\right) & =\frac{1}{2 i \pi} \operatorname{Tr}\left(\int_{\gamma}(\lambda-\tilde{\lambda})^{-s}\left(\Delta_{L}-\lambda\right)^{-1} d \lambda\right), \\
& =\frac{1}{2 i \pi} \operatorname{Tr}\left(\int_{\tilde{c}_{\tilde{\lambda}, \varepsilon}}(\lambda-\tilde{\lambda})^{-s}\left(\Delta_{L}-\lambda\right)^{-1} d \lambda\right),
\end{aligned}
$$

in which $c_{\tilde{\lambda}, \varepsilon}$ denotes the contour obtained by following $c_{\tilde{\lambda}}$ at a distance $\varepsilon$. The second identity comes from Cauchy integral formula since, when $\operatorname{Re}(s)>1$ the contribution of a large circle centered at $\tilde{\lambda}$ tends to zero when the radius grows to infinity.

The same formulas are true for $\Delta_{F}$ and using the fact that $\left(\Delta_{L}-\lambda\right)^{-s}$ and $\left(\Delta_{F}-\lambda\right)^{-s}$ are trace class for $\operatorname{Re} s>1$, we can exchange the contour integration and the trace operation to obtain

$$
\zeta\left(s, \Delta_{L}-\tilde{\lambda}\right)-\zeta\left(s, \Delta_{F}-\tilde{\lambda}\right)=\frac{1}{2 i \pi} \int_{c_{\tilde{\lambda}, \varepsilon}}(\lambda-\tilde{\lambda})^{-s} \operatorname{Tr}\left(\left(\Delta_{L}-\lambda\right)^{-1}-\left(\Delta_{F}-\lambda\right)^{-1}\right) d \lambda
$$


Using prop. 5.3 and the definition of $\tilde{\xi}$ we obtain

$$
\zeta\left(s, \Delta_{L}-\tilde{\lambda}\right)-\zeta\left(s, \Delta_{F}-\tilde{\lambda}\right)=\int_{c_{\lambda, \varepsilon}}(\lambda-\tilde{\lambda})^{-s} \tilde{\xi}^{\prime}(\lambda) d \lambda .
$$

We rewrite the right-hand side in the following form : $\zeta_{1}(s)+\zeta_{2}(s)$ where $\zeta_{1}$ corresponds to the part of the contour $c_{\lambda, \varepsilon}$ that is in the half-plane $\{\operatorname{Re} \lambda \leq-C\}$, and $\zeta_{2}$ is the remaining part of that contour.

The function $\zeta_{2}$ extends to an entire function of $s$ and for $\operatorname{Re}(s)<1$ we may let $\varepsilon$ go to 0 , giving

$$
\forall s, \operatorname{Re}(s)<1, \zeta_{2}(s)=2 i \sin (\pi s) \int_{-C}^{\tilde{\lambda}}(\lambda-\tilde{\lambda})_{0}^{-s} \tilde{\xi}^{\prime}(\lambda) d \lambda,
$$

where the integral is along the part of the cut $c_{\tilde{\lambda}}$ that belongs to the half-plane $\{\operatorname{Re}(\lambda)>-C\}$.

For $\zeta_{1}$ we may first let $\varepsilon$ go to 0 and obtain :

$$
\zeta_{1}(s)=2 i \sin (\pi s) \int_{-\infty}^{-C}(\lambda-\tilde{\lambda})_{0}^{-s} \tilde{\xi}^{\prime}(\lambda) d \lambda .
$$

We make a further reduction by using the following technical lemma.

Lemma 5.8. On $\mathbb{C} \times\{|z|<1\}$, we define $\rho(s, z)=(1-z)^{-s}-1$. For any $r<1$, and any $R>0$, the following holds for any $|z| \leq r$, and any $|s| \leq R$

$$
|\rho(s, z)| \leq \frac{\exp \left(\frac{R r}{1-r}\right)}{1-r} \cdot|s| \cdot|z|
$$

Proof. We start from

$$
\rho(s, z)=\sum_{k \geq 1} \frac{(-s)^{k}[\ln (1-z)]^{k}}{k !} .
$$

By integration we have $|\ln (1-z)| \leq \frac{1}{1-r}|z|$ so that

$$
|\rho(s, z)| \leq \exp \left(\frac{|s||z|}{1-r}\right)-1=\int_{0}^{\frac{|s||z|}{1-r}} \exp (v) d v .
$$

The claim then follows.

For $\operatorname{Re}(\lambda) \leq-C$, there exists some $r<1$ such that $\left|\frac{\tilde{\lambda}}{\lambda}\right| \leq r$. We can thus write

$$
(\lambda-\tilde{\lambda})^{-s}=\lambda^{-s}\left(1+\rho\left(s, \frac{\tilde{\lambda}}{\lambda}\right)\right)
$$

for any $\lambda$ such that $\operatorname{Re}(\lambda) \leq-C$ and $\lambda \notin(-\infty,-C)$.

Fix some $R$, For $s$ such that $\operatorname{Re}(s)>0$ and $|s| \leq R$, using the bound in Lemma 5.8 we may let $\varepsilon$ go to zero and write

where

$$
\zeta_{1}(s)=2 i \sin (\pi s) \int_{-\infty}^{-C}|\lambda|^{-s} \tilde{\xi}^{\prime}(\lambda) d \lambda+2 i \sin (\pi s) \tilde{R}_{C}(s, \tilde{\lambda})
$$

$$
\tilde{R}_{C}(s, \tilde{\lambda})=\int_{-\infty}^{-C}|\lambda|^{-s} \tilde{\xi}^{\prime}(\lambda) \rho\left(s, \frac{\tilde{\lambda}}{\lambda}\right) d \lambda .
$$


Using Lemma 5.8 and Lemma 5.6 we find that, for any extension (regular or not) $\tilde{R}_{C}(\cdot, \lambda)$ can be analytically continued to $\operatorname{Re}(s)>-1$, and that $\tilde{R}_{C}(0)=0$.

Adding up $\zeta_{1}$ and $\zeta_{2}$ we obtain the following proposition.

Proposition 5.9. For any extension, the function $R_{C}(s, \tilde{\lambda})$ which is defined for s large by

$$
R_{C}(s, \tilde{\lambda})=\zeta\left(s, \Delta_{L}-\tilde{\lambda}\right)-\zeta\left(s, \Delta_{F}-\tilde{\lambda}\right)-2 i \sin (\pi s) \int_{-\infty}^{-C}|\lambda|^{-s} \tilde{\xi}^{\prime}(\lambda) d \lambda-\zeta_{2}(s)
$$

can be analytically continued to $R e(s)>-1$ and $R_{C}(s, \tilde{\lambda})$ vanishes at least at second order at $s=0$.

Proof. By inspection and using the definitions of the different functions that appear in the expression of $R_{C}$ we find that

$$
R_{C}(s, \tilde{\lambda})=2 i \sin (\pi s) \tilde{R}_{C}(s, \tilde{\lambda}) .
$$

Using the bounds given by Lemmas 5.6 and 5.8 we find a constant $\tilde{C}$ such that

$$
\forall \lambda<-C,\left.\left.|| \lambda\right|^{-s} \tilde{\xi}^{\prime}(\lambda) \rho\left(s, \frac{\tilde{\lambda}}{\lambda}\right)|\leq \tilde{C}| s|\cdot| \lambda\right|^{-\operatorname{Re}(s)-2},
$$

where $\tilde{C}$ depends on $C, \tilde{\lambda}$ and is uniform for $|s| \leq R$. The claim follows

In particular, in the regular case, we obtain the following corollary (compare with [23])

Corollary 5.10. If $L$ defines a regular extension then $(s-1) \zeta\left(s, \Delta_{L}-\tilde{\lambda}\right)$ extends to a holomorphic function on $\operatorname{Re}(s)>-\beta_{1}$.

Proof. The zeta regularization of the Friedrichs extension is well-known and well studied starting from the small-time asymptotics of the heat kernel (obtained for instance from [7]). The function $(s-1) \zeta\left(\Delta_{F}-\tilde{\lambda}\right)$ is thus known to extends holomorphically to $\mathbb{C}$ (see [2, 3, 17, 19]). Moreover the preceding proposition yields that

$(s-1) \zeta\left(s, \Delta_{L}-\tilde{\lambda}\right)=(s-1) \cdot\left[\zeta\left(s, \Delta_{F}-\tilde{\lambda}\right)+\frac{\sin (\pi s)}{\pi}\left(h_{C}(s)+\frac{\alpha_{0}}{s} \exp (-s \ln C)\right)+\zeta_{2}(s)+R_{C}(s, \tilde{\lambda})\right]$.

The statement thus follows by examining the analytic continuation of each individual term.

Remark 5.11. By evaluating everything at $s=0$ we obtain

$$
\zeta\left(0, \Delta_{L}-\tilde{\lambda}\right)=\zeta\left(0, \Delta_{F}-\tilde{\lambda}\right)+\alpha_{0} .
$$

In the regular case, we can thus define the regularized zeta determinant by the usual formula

$$
\operatorname{det}_{\zeta}\left(\Delta_{L}-\tilde{\lambda}\right)=\exp \left(-\zeta^{\prime}\left(0, \Delta_{L}-\tilde{\lambda}\right)\right)
$$

and we obtain the following theorem.

Theorem 2. Let $L$ define a regular extension and set $\Gamma$ to be

$$
\Gamma=\lim _{\lambda \rightarrow \infty} \ln (D(-|\lambda|))-\alpha_{0} \ln (-|\lambda|) .
$$

Then, for any $\tilde{\lambda} \in \Omega$ we have

$$
\operatorname{det}_{\zeta}\left(\Delta_{L}-\tilde{\lambda}\right)=e^{-\Gamma} \cdot D(\tilde{\lambda}) \cdot \operatorname{det}_{\zeta}\left(\Delta_{F}-\tilde{\lambda}\right) .
$$


Proof. According to the preceding proposition, we have

$$
\zeta^{\prime}\left(0, \Delta_{L}-\tilde{\lambda}\right)-\zeta^{\prime}\left(0, \Delta_{F}-\tilde{\lambda}\right)=\zeta_{2}^{\prime}(0)+h_{C}(0)-\alpha_{0} \ln (C) .
$$

We compute

Combining the two we find

$$
\zeta_{2}^{\prime}(0)=2 i \pi[\tilde{\xi}(\tilde{\lambda})-\tilde{\xi}(-C)]
$$

$$
\begin{aligned}
\zeta^{\prime}\left(0, \Delta_{L}-\tilde{\lambda}\right)-\zeta^{\prime}\left(0, \Delta_{F}-\tilde{\lambda}\right) & =2 i \pi \tilde{\xi}(\tilde{\lambda})-2 i \pi \tilde{\xi}(-C)+h_{C}(0)-\alpha_{0} \ln (C) \\
& =2 i \pi \tilde{\xi}(\tilde{\lambda})+\ln (D(-C))-\alpha_{0} \ln (C)+h_{C}(0)
\end{aligned}
$$

This implies the result with $\Gamma$ replaced by the quantity $\ln (D(-C))-\alpha_{0} \ln (C)+h_{C}(0)$ (which proves in particular that the latter doesn't depend on $C$ large enough). When we let $C$ go to infinity, on the one hand $\ln (D(-C))-\alpha_{0} \ln (C)$ goes to $\Gamma$, and on the other hand, since

$$
h_{C}(0)=\int_{C}^{\infty}\left(2 i \pi \tilde{\xi}^{\prime}(-|\lambda|)-\frac{\alpha_{0}}{\lambda}\right) d \lambda
$$

and the function inside the integral is $L^{1}, h_{C}(0)$ goes to 0 . This finishes proving the theorem.

Remark 5.12. As soon as $h_{C}$ allows the definition of the relative zeta determinant of $\Delta_{L}-\tilde{\lambda}$ and $\Delta_{F}-\tilde{\lambda}$, then, using theorem 2 and differentiating with respect to $\lambda$, we recover a well-known fact of this theory:

$$
\partial_{\tilde{\lambda}}\left(\ln \operatorname{det}\left(\Delta_{L}-\tilde{\lambda}\right)-\ln \operatorname{det}\left(\Delta_{F}-\tilde{\lambda}\right)\right)=2 i \pi \tilde{\xi}^{\prime}(\tilde{\lambda}) .
$$

(compare with [10, 15, 6])

Remark 5.13. For non-regular extensions, it is still possible to analytically continue $\zeta$ to Res $>0$ and to define a zeta-regularized determinant by picking some coefficient in the asymptotic expansion of $\zeta\left(s, \Delta_{L}-\tilde{\lambda}\right)$ near 0 (see [17]). Note however, that the limit $\tilde{\lambda} \rightarrow 0$ will be problematic.

5.4. Proof of theorem 1. In order to get the theorem of the introduction, we now let $\tilde{\lambda}$ go to 0 . We thus modify the zeta-regularized determinant in order to exclude the eigenvalue 0 . Define by $\delta_{L}$ $\left(\right.$ resp. $\left.\delta_{F}\right)$ the dimensions of $\operatorname{ker}\left(\Delta_{L}\right)\left(\right.$ resp. $\operatorname{ker}\left(\Delta_{F}\right)$ ). Equation (5.4) implies that 0 is a pole of $\frac{D^{\prime}}{D}$ with residue $d:=\delta_{L}-\delta_{F}$ so that we can define $D^{*}(0)$

$$
D^{*}(0):=\lim _{\lambda \rightarrow 0} D(\lambda)(-\lambda)^{-\left(\delta_{L}-\delta_{F}\right)} .
$$

On the other hand, we define the modified zeta function by

$$
\zeta^{*}\left(s, \Delta_{F}-\tilde{\lambda}\right)=\zeta\left(s, \Delta_{F}-\tilde{\lambda}\right)-\delta_{F}(-\tilde{\lambda})^{-s}
$$

and the corresponding modified determinant.

Definition 5.14. Let $L$ be defining a regular extension (or $L=F$ ), the modified zeta determinant of $\Delta_{L}$ is defined by

$$
\operatorname{det}_{\zeta}^{*}\left(\Delta_{L}\right)=\lim _{\tilde{\lambda} \rightarrow 0}(-\tilde{\lambda})^{-\delta_{L}} \operatorname{det}_{\zeta}\left(\Delta_{L}-\tilde{\lambda}\right)
$$

Using this definition for $\Delta_{L}$ and $\Delta_{F}$, and plugging into (5.12), the powers of $-\tilde{\lambda}$ cancel out and we may let $\tilde{\lambda}$ go to zero. We thus obtain the theorem in the introduction (Thm. 1).

When $d=0$, the prefactor $D^{*}(0)$ may be computed using the method of section 4.3, When $d>0$ then this method has to be refined to compute more terms in the Taylor expansion of $S(\lambda)$ at $\lambda=0$. In the following example, we will pay special attention to addressing the question of the kernel of $P+Q S(0)$. 
5.5. On the Euclidean sphere with one $4 \pi$ and $\operatorname{six} \pi$ singularities. We consider the Euclidean sphere with six $\pi$ singularities and one $4 \pi$ conical point. We define

$$
A^{ \pm}=\left(\begin{array}{c}
a_{0}^{ \pm} \\
a_{-\frac{1}{2}}^{ \pm} \\
a_{+\frac{1}{2}}^{ \pm} \\
\tilde{A}^{ \pm}
\end{array}\right)
$$

where $a_{i}^{ \pm}, i=-\frac{1}{2}, 0, \frac{1}{2}$ correspond to the $4 \pi$ singularity and $\tilde{A}^{ \pm}$are the coefficients corresponding to the remaining $\operatorname{six} \pi$ singularities. Recall that for each of the latter there are only two coefficients $a_{0}^{ \pm}$.

A regular extension thus relates only the coefficients $a_{ \pm \frac{1}{2}}^{ \pm}$

We define $P_{\theta}$ and $Q_{\theta}$ by

$$
P_{\theta}:=\left(\begin{array}{ccc}
1 & 0 & 0 \\
0 & \cos \theta I_{2} & 0 \\
0 & 0 & I
\end{array}\right), Q_{\theta}:=\left(\begin{array}{ccc}
0 & 0 & 0 \\
0 & \sin \theta I_{2} & 0 \\
0 & 0 & 0
\end{array}\right) .
$$

This choice defines a regular self-adjoint extension (which is, moreover invariant under complex conjugation). We have

$$
D(\lambda)=\operatorname{det}(P+Q S(\lambda))=\operatorname{det}\left(\cos \theta I_{2}+\sin \theta \tilde{S}(\lambda)\right),
$$

where $\tilde{S}$ is the $2 \times 2$ matrix obtained from $S$ by erasing the first row and column (that correspond to $\left.a_{0}^{ \pm}\right)$and all the rows and columns corresponding to $\tilde{A}^{ \pm}$

According to proposition 4.2 , when $\theta \neq 0, \pi$, the asymptotic expansion of $D$ is given by

$$
\begin{aligned}
\ln D(-|\lambda|) & =2|\nu| \ln (|\lambda|)+\ln \left(\left[\frac{\Gamma(1-|\nu|)}{2^{2|\nu|} \Gamma(1+|\nu|)} \sin \theta\right]^{2}\right)+O(1), \\
& =\ln (|\lambda|)+\ln [\sin \theta]^{2}+O(1),
\end{aligned}
$$

since $|\nu|=\frac{1}{2}$.

Finally, we obtain that, for any $\theta \neq 0, \pi$ such that $-\operatorname{cotan}(\theta)$ isn't an eigenvalue of $\tilde{S}(0)$ the following holds :

$$
\operatorname{det}_{\zeta}^{*}\left(\Delta_{L}\right)=\frac{\operatorname{det}\left(\cos \theta I_{2}+\sin \theta \tilde{S}(0)\right)}{\sin ^{2} \theta} \cdot \operatorname{det}_{\zeta}^{*}\left(\Delta_{F}\right)
$$

\section{REFERENCES}

[1] S. Albeverio, F. Gesztesy, R. Hoegh-Krohn and H. Holden. Solvable models in quantum mechanics. AMS Chelsea Publishing, Providence, RI,2005.

[2] E. Aurell and P. Salomonson. On functional determinants of Laplacians in polygons and simplicial complexes. Comm. Math. Phys., 165(2):233-259, 1994.

[3] M. Bordag, S. Dowker and K. Kirsten. Heat-kernels and functional determinants on the generalized cone. Comm. Math. Phys., 182(2):371-393, 1996.

[4] M. Sh. Birman and M. Z. Solomjak. Spectral theory of selfadjoint operators in Hilbert space. Mathematics and its Applications (Soviet Series). D. Reidel Publishing Co., Dordrecht, 1987. Translated from the 1980 Russian original by S. Khrushchëv and V. Peller.

[5] D. Burghelea, L. Friedlander and T. Kappeler T. Meyer-Vietoris type formula for determinants of elliptic differential operators. J. Funct. Anal., 107(1):34-65, 1992.

[6] G. Carron. Déterminant relatif et la fonction Xi. American J. of Math., 124(2):307-352, 2002.

[7] J. Cheeger. Spectral geometry of singular Riemannian spaces. J. Differential Geom., 18(4):575-657, 1983.

[8] J. Cheeger and M. Taylor. On the diffraction of waves by conical singularities. I. Comm. Pure Appl. Math., 35(3):275-331, 1982.

[9] J. Fay. Kernel Functions, Analytic Torsion and Moduli Space. Memoirs of the AMS, 92, 1992. 
[10] R. Forman. Functional determinants and geometry. Invent. Math., 88:447-493, 1987.

[11] G. Forni. Sobolev regularity of solutions of the cohomological equation, preprint, http://arxiv.org/abs/0707.0940v2, 2007.

[12] J. Gil, T. Krainer and G. Mendoza. Dynamics on Grassmannians and resolvents of cone operators. http://arxiv.org/abs/0907.0023v1, 2009.

[13] F. Gesteszy and B. Simon. The xi function. Acta Math., 176:49-71, 1996.

[14] G. Grubb. Distributions and operators, volume 252 of Graduate Texts in Mathematics. Springer, New York, 2009.

[15] A. Hassell and S. Zelditch. Determinants of Laplacians in exterior domains. Int. Math. Res. Not., 18:971-1004, 1999.

[16] L. Hillairet. Contribution of periodic diffractive geodesics. Journal of Functional Analysis,226(1):48-89, 2005.

[17] K. Kirsten, P. Loya, J. Park. Functional determinants for general self-adjoint extensions of Laplace-type operators resulting from the generalized cone. Manuscripta Mathematica, 125:95-126, 2008.

[18] A. Kokotov. Polyhedral surfaces and determinants of Laplacians, to appear in Proceedings of AMS, 2012

[19] A. Kokotov and D. Korotkin. Tau-functions on spaces of abelian differentials and higher genus generalizations of Ray-Singer formula. J. Differential Geom., 82(1):35-100, 2009.

[20] M. Kontsevich and A. Zorich. Connected components of the moduli spaces of Abelian differentials with prescribed singularities. Invent. Math., 153:631-678, 2003.

[21] V. Kostrykin and R. Schrader. Laplacians on metric graphs: eigenvalues, resolvents and semigroups. In Quantum graphs and their applications, volume 415 of Contemp. Math., pages 201-225. Amer. Math. Soc., Providence, RI, 2006.

[22] P. Loya, P. Mcdonald, and J. Park. Zeta Regularized Determinants for Conic Manifolds. Journal of Functional Analysis, 242:195-229, 2007.

[23] E. Mooers. Heat kernel asymptotics on manifolds with conic singularities. J. Anal. Math.,78:1-36, 1999.

[24] S. Nazarov and B. Plamenevsky. Elliptic Problems in Domains with Piecewise Smooth Boundaries, volume 13 of de Gruyter Expositions in Mathematics. Berlin, New York: Walter de Gruyter and Co, 1994.

[25] F.J Olver. Asymptotics and special functions. Academic Press, New York, AKP CLASSICS, AK Peters Ltd,Wellesley, MA, 1997.

[26] M. Reed and B. Simon. Methods of modern mathematical physics. II. Fourier analysis, self-adjointness. Academic Press [Harcourt Brace Jovanovich Publishers], New York, 1975.

[27] M. Reed and B. Simon. Methods of modern mathematical physics. I. Academic Press Inc. [Harcourt Brace Jovanovich Publishers], New York, second edition, 1980. Functional analysis.

[28] M. Troyanov. Les surfaces euclidiennes à singularités coniques. Enseign. Math. (2), 32(1-2):79-94, 1986.

[29] D. Yafaev. Mathematical scattering theory: General theory. Translations of Mathematical Monographs, AMS, 1992.

[30] A. Zorich. Flat surfaces. In Frontiers in number theory, physics, and geometry. I, pages 437-583. Springer, Berlin, 2006.

E-mail address: Luc.Hillairet@math.univ-nantes.fr

UMR CNRS 6629-Université de Nantes, 2 rue de la Houssinière, BP 92 208, F-44 322 Nantes Cedex 3, FRANCE

E-mail address: alexey@mathstat.concordia.ca

Department of Mathematics and Statistics, Concordia University, 1455 de Maisonneuve Blvd. West, Montreal, Quebec H3G 1M8 Canada 